\title{
Duality relations among topological effects in string theory
}

\author{
Edward Witten* \\ Physics Department, California Institute of Technology \\ Pasadena CA 91125, USA, and \\ CIT-USC Center for Theoretical Physics \\ University of Southern California, Los Angeles CA, USA \\ E-mail: witzten@ias.edu
}

ABstract: We explore two different problems in string theory in which duality relates an ordinary $p$-form field in one theory to a self-dual $(p+1)$-form field in another theory. One problem involves comparing D4-branes to M5-branes, and the other involves comparing the Ramond-Ramond forms in type-IIA and type-IIB superstring theory. In each case, a subtle topological effect involving the $p$-form can be recovered from a careful analysis of the quantum mechanics of the self-dual $(p+1)$-form.

KEYwords: Șuperstrings and Heterótic Strings, M-Theory, D-branes, String 'Dualitìi.

${ }^{*}$ On leave from Institute for Advanced Study, Princeton NJ 08540 


\section{Contents}

i1. Introduction

2. Reduction to chiral scalar

3. Quantum mechanics of self-dual $p$-forms

i4. Systematic analysis of type-II case

4.1' Outline

Evaluation of $\Omega(x)$

K-Theory definition of $\Omega(x)$

5. Systematic analysis for M5-brane

15.1 Outline

52 Direct computation

15.3 Comparison to physical definition

\section{Introduction}

The purpose of this paper is to explore how certain relatively subtle topological effects in string theory and M-theory transform into each other under dualities. We will look at two cases that are rather similar and can be treated in rough parallel:

1. The "U(1) gauge field" on the world-volume of a type-II D-brane is actually better described as a $\operatorname{Spin}^{c}$ structure (assuming, as we generally will in the present paper, that the background Neveu-Schwarz three-form field $H$ is topologically trivial). This effect, which first showed up in a detailed example [i]

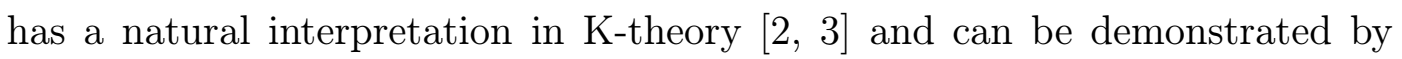
studying global anomalies for elementary strings ending on the D-brane [4]. The effect exists for type-IIA and IIB D $p$-branes for several values of $p$. The problem we will study arises in the case of a type-IIA D4-brane. Such a brane can arise upon compactifying an M5-brane on a circle, in which case the "gauge field" of the D4-brane arises by compactifying the chiral two-form (with selfdual curvature) on the M5-brane. It must somehow be possible to deduce the $\mathrm{Spin}^{c}$ nature of the D4-gauge field from some property of the chiral two-form of the M5-brane. 
2. The Ramond-Ramond four-form field strength $G_{4}$ of type-IIA superstring theory does not, in general, obey conventional Dirac quantization. Under certain

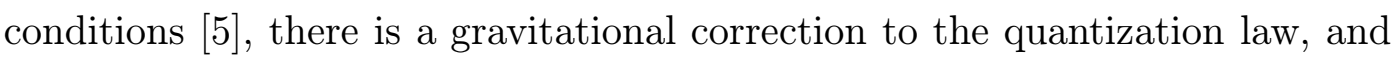
the periods of $G_{4}$ are half-integral. Type-IIA superstring theory on a spacetime $X=\mathbf{S}^{1} \times Y$ is $T$-dual to type IIB on the same spacetime. The $T$-duality maps the relevant part of $G_{4}$ to the self-dual five-form $G_{5}$ of type IIB on $\mathbf{S}^{1} \times Y$. Hence, in this situation, it must be possible to deduce the nonintegrality of the $G_{4}$ periods from some property of the dynamics of $G_{5}$.

What these examples have in common is that on one side of the relation, one considers a field (the "gauge field strength" on the D4-brane, or the four-form of type IIA) whose periods are shifted from conventional Dirac quantization by a gravitational correction. On the other side of the relation is a self-dual Bose field of one degree higher (the three-form of the M5-brane, and the five-form of type IIB) in a related theory. We must somehow deduce the gravitational correction in the lower dimension from the quantum mechanics of the self-dual field in the higher dimension.

The quantum mechanics of a self-dual field is quite subtle and has been studied from many points of view, a sampling being [i, $\overline{0}]-[i 2 \overline{9}]$. Recent work has included construction of brane lagrangians at least locally $[\overline{2} \overline{0}, \overline{2} \overline{4}, \overline{2}, \overline{5}]$ and construction of manifestly supersymmetric and kappa-symmetric equations of motion for multiplets

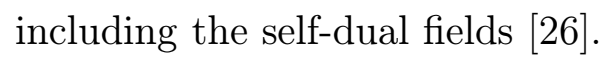

As is most familiar from the case of a chiral scalar (self-dual one-form) in two dimensions, and as we will review in section ${ }_{3-1}^{-1}$ a chiral $p$-form field generally has on a given manifold several possible partition functions, determined by a choice of theta function; one needs a recipe to pick out the right theta function in a given situation. For $p>1$, this has been demonstrated most explicitly in [3] $\left.{ }_{0}^{0}\right]$. The right recipe for picking a theta function depends on some physical input; for the self-dual three-form of the M5-brane, and the self-dual five-form of type IIB, a prescription has been given in [3]1]. For one specific example above two dimensions - the self-dual threeform on $\mathbb{T}^{6}$, where the partition function turns out to be unique (independent of the spin structure on $\mathbb{T}^{6}$ ) - the appropriate partition function has been constructed and

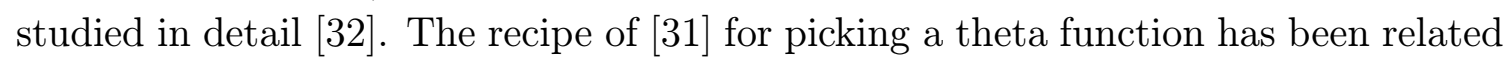

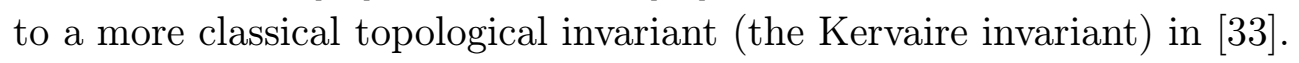

An exception to the statement that the chiral $p$-form has several possible partition functions arises [8-i] if one combines several chiral bosons using an even unimodular lattice. Then one gets complete modular invariance and a unique partition function. This case is very important for the heterotic string [is $\overline{4}$. In a different case (like a single chiral scalar at the free fermion radius, relevant to the present paper), one cannot resolve the ambiguity of the partition function by summing over all possibilities because each candidate partition function has slightly different anomalies, and it does not make sense to add them. In the M-theory and type-IIB applications, 
the chiral $p$-form does not appear by itself but together with additional fields such as fermions. The complete partition function is presumably anomaly-free (this has not been completely demonstrated); anomaly cancellation depends on pairing the proper (spin-structure dependent) partition function of the fermions with the proper partition function of the chiral $p$-form. Thus, one must expect that the recipe for picking a chiral $p$-form partition function depends on the spin structure, and this is the case for the proposal in [31. Once the anomalies are all canceled, it is possible, and perhaps correct physically, to sum over spin structures.

The main goal of the present paper is to show how the quantum mechanics of the self-dual fields gives rise, after compactification on a circle, to the effects mentioned in (1) and (2) above. In section 2 which detailed general theory is not needed. In the rest of the paper, we proceed more systematically. In section 3

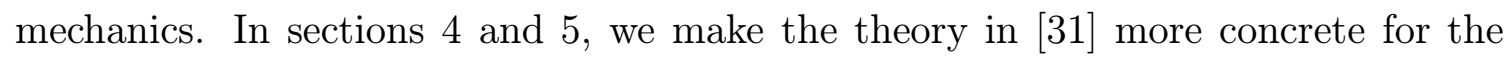
situation of interest and use it to deduce what we need.

The first of our two problems described above is somewhat reminiscent of the problem of relating the mechanism of M5-brane normal bundle anomaly cancella-

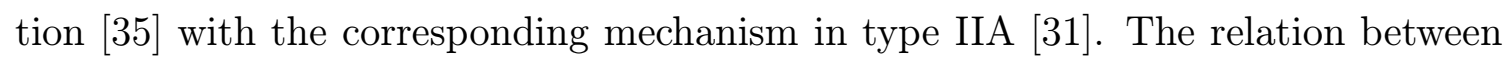

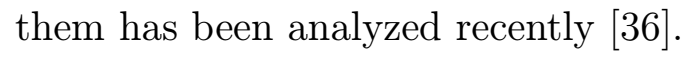

\section{Reduction to chiral scalar}

The goal in the present section is to verify that the phenomena mentioned in the introduction work out correctly in some simple cases in which we can do this without many technicalities. This will perhaps satisfy the curiosity of some readers, and may give others the courage needed to persevere through the technicalities of the rest of the paper.

M5-Brane wrapped on a circle. We first consider the relation of the M5-brane to the D4-brane. Our goal is to analyze the M5-brane on a world-volume $V=$ $S \times R$, where $R$ is an oriented five-manifold and $S$ is a circle with a supersymmetrypreserving spin structure. To do this in general will be the goal of section '5, but things are much simpler in the case $R=\widetilde{S} \times R^{\prime}$, with $\widetilde{S}$ another circle and $R^{\prime}$ a fourmanifold. The simplicity will arise because in this special case, we do not need to understand chiral $p$-forms fields of $p>0$; we can deduce what we need from familiar (though subtle) facts about chiral scalars.

Though we could treat an arbitrary $R^{\prime}$, it will suffice for illustration to take $R^{\prime}=\mathbb{C P}^{2}$. Thus, the fivebrane world-volume will be $V=\Sigma \times \mathbb{C P}^{2}$ where $\Sigma=S \times \widetilde{S}$ is a product of circles; the spin structure on $S$ preserves supersymmetry but either choice may be made on $\widetilde{S}$. The non-trivial cohomology group of $\mathbb{C P}^{2}$ (apart from 
dimensions zero and four) is

$$
H^{2}\left(\mathbb{C P}^{2} ; \mathbb{Z}\right)=\mathbb{Z}
$$

The generator of $H^{2}\left(\mathbb{C P}^{2} ; \mathbb{Z}\right)$ is a self-dual form $\omega$ that obeys

$$
\int_{\mathbb{C P}^{1}} \omega=1, \quad \int_{\mathbb{C P}^{2}} \omega \wedge \omega=1 .
$$

Here $\mathbb{C P}^{1}$ is a linearly embedded subspace of $\mathbb{C P}^{2}$ and generates $H_{2}\left(\mathbb{C P}^{2} ; \mathbb{Z}\right)$.

We suppose that the M-theory spacetime is $X=\Sigma \times C$, where $C$ is a ninedimensional spin-manifold in which $\mathbb{C P}^{2}$ is embedded. M-theory on this spacetime is equivalent to type IIA on $X^{\prime}=\widetilde{S} \times C$; the M5-brane corresponds to a D4-brane wrapped on $R=\widetilde{S} \times \mathbb{C P}^{2}$. $R$ is not a spin manifold, since $\mathbb{C P}^{2}$ is not. As a result, according to [ind, the field strength $F$ of the "U(1) gauge field" on the D4-brane does not obey conventional Dirac quantization. Rather,

$$
\int_{\mathbb{C P}^{1}} \frac{F}{2 \pi}=n+\frac{1}{2}
$$

with integer $n$.

The gauge field on the D4-brane arises by dimensional reduction from the chiral two-form $b$ on the M5-brane. We want to know how (2.3) arises from the theory of a chiral two-form. We consider a limit in which the radii of $S$ and $\widetilde{S}$ are much greater than the size of the $\mathbb{C P}^{2}$. In this case, the physics on the M5-brane reduces to an effective two-dimensional theory on $\Sigma=S \times \widetilde{S}$. In fact, the field $b$ reduces (by the ansatz $b=\omega \phi)$ to a chiral scalar $\phi$ in two dimensions. $\phi$ appears at the self-dual or free fermion radius; ${ }^{1}$ the $\phi$ field is hence equivalent quantum mechanically to a complex fermion $\psi$ of positive chirality.

The $\psi$ field propagates on the Riemann surface $\Sigma$, and the partition function of $\psi$ depends on a choice of spin structure on $\Sigma$. So to describe the physics, we need to know the effective spin structure on $\Sigma$ in the low energy theory, given the underlying choice of spin structure on the M-theory spacetime $X=\Sigma \times C$. Since choosing a spin structure on $X$ is equivalent to choosing a spin structure on $\Sigma$ and choosing one on $C$, in the microscopic M-theory description a spin structure was chosen on $\Sigma$ at the beginning. In fact, as we noted above, we are interested in the case that this spin structure is the product of the supersymmetric spin structure on $S$ and any desired

\footnotetext{
${ }^{1}$ In general, if the M5-brane is compactified to two dimensions on a four-manifold $R^{\prime}$, the chiral two-form reduces to a set of two-dimensional scalars with momentum lattice given by the twodimensional cohomology lattice of $R^{\prime}$. For $R^{\prime}=\mathbb{T}^{4}$, this assertion is built into the detailed computation in [32]. For $R^{\prime}=\mathbb{C P}^{2}$, the lattice is one-dimensional, generated by a vector $\omega$ with $\omega^{2}=1$; this is the lattice of a chiral boson with the free-fermion radius. (Depending on how $R^{\prime}$ is embedded in the full spacetime, some of the conservation laws associated with the momentum lattice may be violated by instantons constructed from membranes with boundary on $R^{\prime}$. This phenomenon is irrelevant for determining the fivebrane partition function in the large volume limit.)
} 
spin structure on $\widetilde{S}$. It is natural to guess that the effective spin structure on $\Sigma$ in the low energy theory is just the spin structure on $\Sigma$ that we start with microscopically. This assertion almost follows just from the fact that the map from the microscopic to the macroscopic spin structure must be invariant under the action of $\operatorname{SL}(2, \mathbb{Z})$ on $\Sigma$, and can be verified using the techniques of sections 自 and

In the theory of a D4-brane on $\widetilde{S} \times \mathbb{C P}^{2}$, with $\widetilde{S}$ regarded as the "time" direction, the flux $\left(2.3_{3}\right)$ can be interpreted as a conserved charge. Going back to the self-dual three-form theory on the M5-brane worldvolume $V=S \times \widetilde{S} \times \mathbb{C P}^{2}$, this flux is interpreted as the integral of the self-dual three-form $T$ (which is the curvature of the chiral two-form $b$, defined by $T=d b$ ) over $S \times \mathbb{C P}^{1}$. In terms of the ansatz $b=\omega \phi$, we have $T=\omega \wedge d \phi$, and the conserved charge is

$$
q=\int_{S \times \mathbb{C P}^{2}} \frac{\omega \wedge d \phi}{2 \pi}=\oint_{S} \frac{d \phi}{2 \pi} .
$$

In the free fermion description, $d \phi / 2 \pi$ becomes $\bar{\psi} \psi$ and the charge is the conserved fermion number

$$
q=\oint_{S} \bar{\psi} \psi
$$

Now, since the fermions on $S$ are in the supersymmetric spin structure, both $\psi$ and $\bar{\psi}$ have a single zero mode on $S$. The quantization of the zero modes gives rise, in a way that is familiar from the Ramond sector of superstrings, to a two-fold degeneracy of the ground state. The ground states have fermion number $q= \pm 1 / 2$, and all excited states have half-integral eigenvalues of $q$. Since $q$ is interpreted in the typeIIA description as the flux in (……… $)$, we have explained the half-integrality of that flux starting with the theory of the self-dual three-form on the M5-brane.

It is also instructive to consider, in a similar fashion, a case in which the D4brane is wrapped on a five-manifold $R$ that does not have a $\operatorname{Spin}^{c}$ structure, so that the theory should be inconsistent. Such a case is obtained by taking $R$ to be not a product $\widetilde{S} \times \mathbb{C P}^{2}$ but a $\mathbb{C P}^{2}$ bundle over $\widetilde{S}$ in which the fiber undergoes complex conjugation in going around $\widetilde{S}$. Complex conjugation reverses the sign of $\omega$ and so acts on $\phi$ by $\phi \rightarrow-\phi$. The periods of $\phi$ thus must change sign in going around $\widetilde{S}$, but since they are half-integral, this is impossible. This is the inconsistency. But what does it look like in the free fermion description? From this point of view, $\phi \rightarrow-\phi$ is $\psi \leftrightarrow \bar{\psi}$. Alternatively, if $\psi=\left(\psi_{1}+i \psi_{2}\right) / \sqrt{2}$ with Majorana-Weyl fermions $\psi_{1}, \psi_{2}$, it is

$$
\psi_{1} \longrightarrow \psi_{1}, \quad \psi_{2} \longrightarrow-\psi_{2} .
$$

Both $\psi_{1}$ and $\psi_{2}$ couple to the supersymmetric spin structure on $S$, and in view of $\left(\underline{2} . \overline{6}_{1}^{\prime}\right)$, they see opposite spin structures on $\widetilde{S}$. So $\psi_{1}$ and $\psi_{2}$ together have precisely one zero mode on $S \times \widetilde{S}$. Having an odd number of fermion zero modes means that the partition function vanishes, and that this vanishing cannot be lifted by insertions of local operators (a fermionic operator will not have an expectation value once we 
average over spatial rotations). It should be interpreted as a kind of global anomaly. We will argue in section whenever $R$ is not $\operatorname{Spin}^{c}$.

Analog for type IIB. Now let us briefly discuss the analogous issues in the other case mentioned in the introduction.

Our goal is to compare topological effects in type-IIB and type-IIA superstring theory on $S \times Y$, with $S$ a circle and $Y$ a nine-dimensional spin manifold. But a shortcut along the above lines is possible for the special case $Y=\widetilde{S} \times Y^{\prime}$, with $\widetilde{S}$ another circle and $Y^{\prime}$ an eight-dimensional spin manifold. So we consider type-IIB superstring theory on $S \times \widetilde{S} \times Y^{\prime}$, with the supersymmetric spin structure on the first factor.

For illustration, we consider the case that $Y^{\prime}=\mathbf{H P}^{2}$. The only non-trivial cohomology group of this manifold is $H^{4}\left(\mathbf{H P}^{2} ; \mathbb{Z}\right)=\mathbb{Z}$. The generator is a self-dual four-form $\omega$ such that

$$
\int_{\mathbf{H P}^{1}} \omega=1, \quad \int_{\mathbf{H P}^{2}} \omega \wedge \omega=1
$$

Here $\mathbf{H} \mathbf{P}^{1}$ is a linearly embedded subspace of $\mathbf{H P}^{2}$ and generates $H_{4}\left(\mathbf{H P}^{2} ; \mathbb{Z}\right) . \mathbf{H P}^{2}$ is a spin manifold, so its first Pontryagin class $p_{1}$ is divisible by 2 , and $\lambda=p_{1} / 2$ obeys

$$
\int_{\mathbf{H P}^{1}} \lambda=1
$$

In fact, $\lambda$ is just $\omega$.

We can repeat much of what we have already seen. In compactification on $S \times \widetilde{S} \times \mathbf{H P}^{2}$, with the last factor much smaller than the first two, the chiral fourform $C_{4}$ of type-IIB superstring theory reduces at long distances (via an ansatz $\left.C_{4}=\omega \phi\right)$ to a chiral scalar $\phi$ on $S \times \widetilde{S}$. $\phi$ can be expressed in terms of free fermions, and by the same reasoning as above, if we regard $\widetilde{S}$ as the "time" direction, then the conserved charge

$$
q=\oint_{S} \frac{d \phi}{2 \pi}
$$

takes half-integral values. One can think of $q$ more microscopically as

$$
q=\oint_{S \times \mathbf{H P}} \frac{G_{5}}{2 \pi}
$$

where $G_{5}=d C_{4}$ is the gauge-invariant self-dual five-form of type IIB.

Now we consider a $T$-duality transformation on the first circle $S$. This maps type-IIB superstring theory to type IIA, and the modes of $G_{5}$ that appear in the integral in (12.10') are mapped to $G_{4}$, the Ramond-Ramond four-form field strength of type IIA. In the type-IIA description, $q$ becomes

$$
q=\int_{\mathbf{H P}^{1}} \frac{G_{4}}{2 \pi}
$$


Thus, to account for the half-integrality of $q$ from the type-IIA point of view, we must explain why $G_{4}$ has half-integral periods in this situation.

But this is a consequence of (2.

$$
\int_{U} \frac{G_{4}}{2 \pi}=\frac{1}{2} \int_{U} \lambda+\text { integer }
$$

for any four-cycle $U$ in a type-IIA spacetime. In view of (2. $\left.\overline{2}_{1}^{\prime}\right)$, this is equivalent to half-integrality of $q$. Thus, we have succeeded, in this situation, in reconciling the gravitational shift in the quantization law of the four-form in type IIA with the subtleties of the self-dual five-form of type IIB.

For a more complete study of these problems, where we compactify on only one circle and not two, we need to delve into the theory of chiral $p$-form fields for $p>0$. This will be the subject of sections 垈 and additional aspects of the quantum mechanics of self-dual $p$-forms, starting with the one-form case.

\section{Quantum mechanics of self-dual $p$-forms}

Before looking at our specific problem, we need some more background on chiral $p$ forms.

In constructing the quantum mechanics of an ordinary (not self-dual) $p$-form field on a manifold $M$, one sums over all periods in $H^{p}(M ; \mathbb{Z})$. That is not so for a self-dual $p$-form.

In fact, it is impossible to impose any classical quantization law at all on the periods of a self-dual $p$-form. To illustrate this, let $\Sigma$ be a two-torus constructed as $\mathbb{C} / \Lambda$, where $\mathbb{C}$ is the complex $z$-plane, and $\Lambda$ is a lattice generated by complex numbers 1 and $\tau$ (with $\operatorname{Im} \tau>0$ ). Let $A$ be a cycle in $\Sigma$ that lifts in $\mathbb{C}$ to a path from 0 to 1 , and let $B$ be a cycle that lifts to a path from 0 to $\tau$. Let $\lambda$ be a self-dual one-form. Then $\lambda=c d z$ for some complex constant $c$. If we want, for example, $\int_{A} \lambda / 2 \pi$ to be integral, we need $c \in 2 \pi \mathbb{Z}$, while requiring $\int_{B} \lambda / 2 \pi$ to be integral puts an entirely different condition on $c$.

What happens instead is that a self-dual $p$-form must be treated quantum mechanically; one cannot treat its periods classically. The partition function of such a field is written as a sum over only half the periods. For illustration, let us consider an example [is that is extremely important in string theory: a collection of $8 k$ chiral bosons $\phi_{i}$ in two dimensions, for some integer $k$, associated with an even unimodular lattice $\Gamma$ with positive definite intersection form $($,$) . We set \lambda_{i}=d \phi_{i}$. The partition function in genus one is as follows. Let $\Sigma$ be as above and $q=\exp (2 \pi i \tau)$. Then the partition function of the chiral boson theory on $\Sigma$ is

$$
Z(q)=\frac{\sum_{w \in \Gamma} q^{(w, w) / 2}}{\eta(q)^{8 k}}
$$


with $\eta$ the Dedekind eta-function. In this formula, the partition function is constructed as a sum over a single set of periods - the periods $w_{i}=\int_{A} \lambda_{i} / 2 \pi$, which are the components of a single lattice vector $w \in \Gamma$. In a hamiltonian framework with $A$ regarded as the spatial cycle and $B$ as time, the $A$-periods label the winding (or by self-duality the momentum) states; the theta function in the numerator of (13.11) comes from the sum over these states. Of course, the choice of the particular cycle $A$ is not uniquely determined. The partition function is $\mathrm{SL}(2, \mathbb{Z})$-invariant; by an $\mathrm{SL}(2, \mathbb{Z})$ transformation, one could replace the cycle $A$ by $n A+m B$ for any relatively prime integers $n, m$.

Intuitively, we may think of two periods $\int_{A} \lambda$ and $\int_{A^{\prime}} \lambda$ as commuting if and only if the intersection number $A \cap A^{\prime}$ is zero. There is no way to simultaneously measure non-commuting periods. The partition function is constructed as a sum over a maximal set of commuting periods.

The example relevant to the present paper is slightly more subtle: it is the case that the chiral bosons $\phi_{i}$ are derived from a lattice $\Gamma$ that is unimodular, but not even. In fact, the prototype for us is a single chiral boson at the free fermion radius, that is to say $\Gamma$ is a one-dimensional lattice generated by a vector $\omega$ with $(\omega, \omega)=1$. In this case, there is not a single partition function; rather (as is apparent from the description by free fermions) there is a partition function for each choice of spin structure. It is instructive to examine these partition functions. They are conveniently written in terms of standard functions as

$$
Z\left[\begin{array}{l}
\theta \\
\phi
\end{array}\right](z \mid \tau)=\frac{\vartheta\left[\begin{array}{l}
\theta \\
\phi
\end{array}\right](z \mid \tau)}{\eta(\tau)}
$$

where $\theta$ and $\phi$ are 0 and $1 / 2$ and $z$ is an extra variable included to represent the coupling to a background gauge field. The partition function in the absence of this field is obtained by setting $z=0$. The functions in the numerator on the right-hand side are called theta functions with characteristics. They are explicitly

$$
\begin{aligned}
\vartheta\left[\begin{array}{l}
0 \\
0
\end{array}\right](z \mid \tau) & =\sum_{n \in \mathbb{Z}} q^{n^{2} / 2} \exp (2 \pi i n z) \\
\vartheta\left[\begin{array}{c}
0 \\
1 / 2
\end{array}\right](z \mid \tau) & =\sum_{n \in \mathbb{Z}}(-1)^{n} q^{n^{2} / 2} \exp (2 \pi i n z) \\
\vartheta\left[\begin{array}{c}
1 / 2 \\
0
\end{array}\right](z \mid \tau) & =\sum_{n \in \mathbb{Z}+1 / 2} q^{n^{2} / 2} \exp (2 \pi i n z) \\
\vartheta\left[\begin{array}{c}
1 / 2 \\
1 / 2
\end{array}\right](z \mid \tau) & =i \sum_{n \in \mathbb{Z}+1 / 2}(-1)^{n+1 / 2} q^{n^{2} / 2} \exp (2 \pi i n z) .
\end{aligned}
$$

We have written these theta functions as sums over the $A$-period $n=\int_{A} d \phi / 2 \pi$. By $\operatorname{SL}(2, \mathbb{Z})$, one could instead write each of these theta functions as a sum over 
any other chosen period of $d \phi$. While $\vartheta\left[\begin{array}{l}1 / 2 \\ 1 / 2\end{array}\right]$, which corresponds to the odd spin structure, is $\mathrm{SL}(2, \mathbb{Z}$ )-invariant (up to a $c$-number multiple that reflects the modular weight plus an anomalous phase), the others are permuted by $\operatorname{SL}(2, \mathbb{Z})$, so if one chooses to write $\vartheta\left[\begin{array}{l}0 \\ 0\end{array}\right]$, for example, with a different choice of the period, one might have to use the formula for $\vartheta\left[\begin{array}{c}1 / 2 \\ 0\end{array}\right]$.

In constructing the theta function as a sum over the values of the $A$-period $n$, this period is integral for $\theta=0$ and half-integral for $\theta=1 / 2$. Therefore the answer to the question of whether a given period of the self-dual one-form is integral or halfintegral depends on the choice of theta function. On the other hand, $\phi$ determines the sign factors in the sum over the $A$-periods. A configuration with a given value of the $A$-period $n$ is weighted by a sign +1 if $\phi=0$ and by a sign $(-1)^{n}$ (or $(-1)^{n+1 / 2}$ if $n$ is half-integral) if $\phi=1 / 2$.

Now, we want to describe the theta functions in a way that generalizes to higher genus surfaces and also to self-dual $p$-forms of $p>1$. We will define a $\mathbb{Z}_{2}$-valued function $\Omega(x)$ on the lattice $\Lambda$ as follows. ${ }^{2}$ For the lattice points 1 and $\tau$, we set

$$
\Omega(1)=(-1)^{2 \phi}, \quad \Omega(\tau)=(-1)^{2 \theta} .
$$

We extend $\Omega$ to a function on the whole lattice by requiring

$$
\Omega(x+y)=\Omega(x) \Omega(y)(-1)^{(x, y)},
$$

where $(x, y)=-(y, x)$ is the intersection form on the lattice $\Lambda$. For example, this definition gives

$$
\Omega(1+\tau)=-\Omega(1) \Omega(\tau),
$$

since 1 and $\tau$ correspond to the cycles $A$ and $B$, whose intersection number is 1 . ( is the basic formula. Theta functions are in natural one-to-one correspondence with $\mathbb{Z}_{2}$-valued functions on the lattice that obey this relation. Given $\Omega$, the characteristics $\theta, \phi$ are extracted from (3. functions that we gave above.

Let $\Lambda_{1}$ and $\Lambda_{2}$ be, respectively, the sublattices of $\Lambda$ generated by 1 and by $\tau$; we call these the $A$-lattice and the $B$-lattice. As we saw above, a configuration with $A$-period $n$ contributes to the theta function (in the representation of that function as a sum over the $A$-periods) with a sign 1 or $(-1)^{n}$ depending on $\phi$. (…4. that $\Omega(x)$ for $x$ in the $A$-lattice is simply the sign factor with which a configuration of $A$-period $n=x$ (or $n=x+1 / 2$ ) contributes to the theta function. Likewise, we saw above that $\theta$ determines whether the $A$-periods are integral or half-integral, and thus this is determined by $\Omega(x)$ for $x$ in the $B$-lattice.

The classification of theta functions by $\mathbb{Z}_{2}$-valued functions $\Omega(x)$ extends beyond the genus one case that we have just considered: level one theta functions of any

\footnotetext{
${ }^{2}$ In $[B \overline{1} \bar{i}$, , this function was called $H(x)$, but I want to avoid notational clashes with the three-form field $H$ of string theory and $H^{i}(M)$ for cohomology groups.
} 
lattice $\Lambda$ with unimodular antisymmetric form ( , ) and a metric for which this form is positive and of type $(1,1)$ are classified by functions $\Omega$ obeying ( $(1 . \overline{3} . \overline{1})$. This fact has a differential-geometric explanation that was reviewed in [isin. (The basic idea is that such an $\Omega$ determines a line bundle over $\Sigma$; this line bundle has up to constant multiples a unique holomorphic section which is the theta function.) For our present purposes, we will simply note that the functions $\Omega$ that obey (13.5. $\operatorname{SL}(2, \mathbb{Z})$ the same way that theta functions do. In this assertion, the sign factor $(-1)^{(x, y)}$ in $\left(\begin{array}{l}3 \\ .\end{array}\right)$ is essential. For example, the theta function $\vartheta\left[\begin{array}{l}1 / 2 \\ 1 / 2\end{array}\right]$ associated with the odd spin structure is $\mathrm{SL}(2, \mathbb{Z})$-invariant, so it must be associated with a function $\Omega(x)$ that is likewise $\operatorname{SL}(2, \mathbb{Z})$-invariant. Since $\theta=\phi=1 / 2$, this theta function has $\Omega(1)=\Omega(\tau)=-1$. As $\operatorname{SL}(2, \mathbb{Z})$ can map the lattice points 1 or $\tau$ to $1+\tau$, it follows that $\Omega(1+\tau)$ must equal -1 , which is what we get from (3.6.

To write the four theta functions by explicit formulas as in $\left(\underline{3} . \overline{3} \overline{3}_{1}\right)$ requires a choice of $A$-lattice. Some more information is needed, though, because the choice of $A$-lattice is invariant under $\tau \rightarrow \tau+1$, but this operation permutes the theta functions in a non-trivial fashion. If one is also given a choice of $B$-lattice (and thus essentially the basis $(1, \tau)$ for the lattice $\Lambda$ ), this is more than enough information to enable the writing of the explicit formulas in ( the $B$-cycles mod 2.) If one has chosen both the $A$-lattice and the $B$-lattice, then one has an explicit $\mathrm{SL}(2, \mathbb{Z})$ transformation $\tau \rightarrow-1 / \tau$ that exchanges them. It exchanges $\theta$ and $\phi$, and thus exchanges a half-integral shift in the value of the $A$-period $n$ with a sign factor by which the different values of the $A$-period are weighted.

Generalization. Now let us consider the generalization to a self-dual $p$-form field $G_{p}$, of $p$ possibly bigger than 1 , on a $2 p$-dimensional manifold $M$. (For a detailed treatment via holomorphic factorization of the partition function of a non-chiral theory, see $[\underline{\underline{\beta}} \overline{\underline{0}} \overline{1}]$.) The periods take values in $\Lambda=H^{p}(M ; \mathbb{Z})$, which for simplicity we will assume to be torsion-free. Thus $\Lambda$ is a lattice, with an antisymmetric bilinear form ( , ) of determinant 1 that is given by the intersection pairing on $M$. If $\Lambda$ has rank $2 g$, then it has $2^{2 g}$ distinguished theta functions $\vartheta\left[\begin{array}{l}\theta \\ \phi\end{array}\right](z \mid \tau)$ that we will introduce momentarily. The partition function of $G_{p}$ is $\vartheta\left[\begin{array}{l}\theta \\ \phi\end{array}\right](z \mid \tau) / \Delta$, where $\Delta$ (analogous to $\eta(\tau)$ in $\left(\begin{array}{l}3.2 \\ 2\end{array}\right)$ ) is uniquely determined from the non-zero modes of $G$. The subtlety comes from the choice of theta function in the numerator.

As in the case of a one-form field, the periods of $G$ are not all simultaneously measurable. The best that one can do is to pick a maximal sublattice $\Lambda_{1}$ consisting of mutually "commuting" periods. $\Lambda_{1}$ is a lattice of $A$-periods, that is, it is a halfdimensional sublattice of $\Lambda$ such that $(x, y)=0$ for $x, y \in \Lambda_{1}$. It is convenient, though not necessary, to pick also a complementary lattice $\Lambda_{2}$ of $B$-periods. Thus, $\Lambda=\Lambda_{1} \oplus \Lambda_{2}$, and $(x, y)=0$ for $x, y \in \Lambda_{2}$. Picking the $B$-periods and $A$-periods gives an explicit period matrix $\tau_{i j}=\tau_{j i}, i, j=1, \ldots, g$ for the lattice $\Lambda$. 
Once the $A$-cycles and $B$-cycles are fixed, one can write an explicit formula for the theta functions. One picks a half-lattice vector $\theta \in \frac{1}{2} \Lambda_{1} / \Lambda_{1}$, and a half-lattice vector $\phi \in \frac{1}{2} \Lambda_{2} / \Lambda_{2}$. The theta function with characteristics $\theta, \phi$ is then

$$
\vartheta\left[\begin{array}{l}
\theta \\
\phi
\end{array}\right]\left(z_{i} \mid \tau\right)=\sum_{n \in \Lambda_{1}+\theta} \exp \left(i \pi \sum_{i j} n^{i} n^{j} \tau_{i j}+2 \pi i n^{i}\left(z_{i}+\phi_{i}\right)\right) .
$$

The $z_{i}$ are parameters that measure the coupling to a background $p$-form potential; the partition function is obtained by setting $z_{i}=0$ (and dividing by $\Delta$ ).

From ( $\left(\overline{3}_{1} . \overline{7}_{1}\right)$, we see that if we write the theta function as a sum over $A$-periods, then the $A$-periods are shifted from integers by $\theta \in \frac{1}{2} \Lambda_{1} / \Lambda_{1}$. But the sign factor in the sum over $A$-periods is determined by $\phi$.

As in the $g=1$ case that we discussed first, the theta functions are most naturally classified by a $\mathbb{Z}_{2}$-valued function $\Omega(x)$ on the lattice $\Lambda$ that obeys the fundamental relation

$$
\Omega(x+y)=\Omega(x) \Omega(y)(-1)^{(x, y)} \quad \text { for all } x, y \in \Lambda .
$$

Given such a function, one defines the characteristics $\theta, \phi$ by

$$
\begin{array}{ll}
\Omega(x)=(-1)^{2(x, \phi)} & \text { if } x \in \Lambda_{1}, \\
\Omega(x)=(-1)^{2(x, \theta)} & \text { if } x \in \Lambda_{2},
\end{array}
$$

and then the theta function can be defined by the formula in ( $\left.\overline{1}_{3} . \overline{7}_{1}\right)$. As mentioned above, there is also a more intrinsic procedure to go from $\Omega$ to the theta function (use $\Omega$ to construct a line bundle and take its holomorphic section).

Combining the above definitions, we can see how the theta function depends on $\Omega$. For $x \in \Lambda_{1}, \Omega(x)$ is a sign factor in the sum over $A$-periods, and for $x \in \Lambda_{2}$, $\Omega(x)$ controls the nonintegrality of the $A$-periods. This generalizes what we explained above for $g=1$.

Specializing to $M=S \times F$. In general, this formalism is somewhat abstract, partly because for a general $2 p$-dimensional manifold $M$, there is no particularly nice choice of $A$-periods and $B$-periods. Nice choices do exist if $M=S \times F$, with $S$ a circle and $F$ a manifold of dimension $2 p-1$. This case is our focus in the present paper. The theory of a self-dual $p$-form $G_{p}$ on such an $M$ reduces at low energies on $F$ to a theory of an ordinary $p$-form $G_{p}^{\prime}$ with no self-duality, or (after a duality transformation) to a theory of an ordinary $(p-1)$-form $G_{p-1}^{\prime}$. Correspondingly, the cohomology of $M$ splits as

$$
\begin{aligned}
H^{p}(M ; \mathbb{Z}) & =H^{p}(F ; \mathbb{Z}) \oplus H^{1}(S ; \mathbb{Z}) \otimes_{\mathbb{Z}} H^{p-1}(F ; \mathbb{Z})= \\
& =H^{p}(F ; \mathbb{Z}) \oplus H^{p-1}(F ; \mathbb{Z}) .
\end{aligned}
$$

We take $\Lambda=H^{p}(M ; \mathbb{Z})$, so that a partition function of $G_{p}$ on $M$ is a theta function of $\Lambda$, and we set $\Lambda_{1}=H^{p}(F ; \mathbb{Z}), \Lambda_{2}=H^{p-1}(F ; \mathbb{Z})$. 
We take the lattice of $A$-periods to be $\Lambda_{1}$ and the lattice of $B$-periods to be $\Lambda_{2}$. A theta function for $\Lambda$ can be constructed either as a sum over $A$-periods corresponding to the representation of the theory on $F$ in terms of $G_{p}^{\prime}$ - or as a sum over $B$-periods - corresponding to the representation of the theory on $F$ in terms of $G_{p-1}^{\prime}$.

No matter which representation one uses, the theta function of $\Lambda$ is determined by a choice of a suitable function $\Omega(x)$ on $\Lambda$. How to construct such a function for

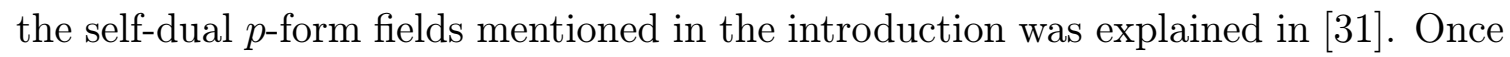
$\Omega$ is selected, its restriction to $\Lambda_{1}$ determines a sign factor in the sum over periods if one uses the description of the theory in terms of $G_{p}^{\prime}$, or a nonintegrality of the $G_{p-1}^{\prime}$ periods in the other description. Conversely, the restriction of $\Omega$ to $\Lambda_{2}$ determines the nonintegrality of $G_{p}^{\prime}$ periods, or a sign factor in the sum over $G_{p-1}^{\prime}$.

The fact that a sign factor on one side becomes, after duality, a nonintegrality of the periods in the other description can also be explained more microscopically in terms of a Feynman path integral representation of the $G_{p}^{\prime}$ and $G_{p-1}^{\prime}$ theories. More generally, in $d$ dimensions, a phase factor in a theory with $k$-form curvature $G_{k}$ is always translated after duality to a shift in periods of a dual field $G_{d-k}$. This holds for all $d$ and $k$. A path integral derivation of this fact can be found (for $d=4, k=2$, but the general case is not essentially different) in [i $3 \overline{3} \overline{-1}$, section 4.2$]$.

\section{Systematic analysis of type-II case}

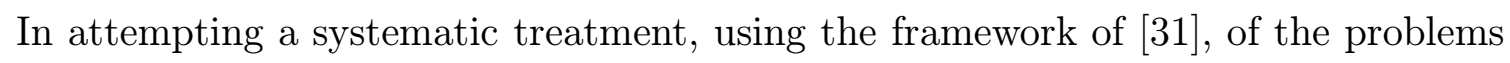
mentioned in the introduction, we will begin with the second problem - understanding the shifted quantization law of the type-IIA four-form from the quantum mechanics of the self-dual five-form of type IIB. This case involves fewer technicalities.

\subsection{Outline}

In type-IIB theory on a ten-dimensional spin manifold $X$, we have a four-form potential $C_{4}$ with a self-dual curvature five-form $G_{5}$. If we could omit the self-duality condition, and we impose conventional Dirac quantization, then the $C_{4}$-fields are classified topologically by a class $x \in H^{5}(X ; \mathbb{Z})$. Here $x$ is represented in de Rham cohomology by $G_{5} / 2 \pi$. We sometimes write informally $x=\left[G_{5} / 2 \pi\right]$.

For an ordinary four-form field, we would construct the partition function by summing over all choices of $x$ (and for each choice of $x$, integrating over all possibilities for $C_{4}$ ). For a four-form of self-dual curvature, we do not sum independently over all values of $x$. Rather, as discussed in section $\underline{3}_{-1}^{-}$above and in $[\overline{3} \overline{1} \overline{1}$, we construct the partition function in terms of a theta function on $\mathbb{T}=H^{5}(X ; \mathrm{U}(1))$, which, if there is no torsion in the cohomology of $X$, is the torus $H^{5}(X ; \mathbb{R}) / H^{5}(X ; \mathbb{Z})$. The theta 
function, as we discussed in section $\underline{\underline{B}}_{-0}^{-}$is constructed by summing over a maximal set of "commuting" periods. ${ }^{3}$

To construct the theta function, as explained in [3i function $\Omega(x)$ from $H^{5}(X ; \mathbb{Z})$ to the group $\mathbb{Z}_{2}=\{ \pm 1\}$, obeying

$$
\Omega(x+y)=\Omega(x) \Omega(y)(-1)^{x \cdot y}
$$

for all $x, y \in H^{5}(X ; \mathbb{Z})$. Here $x \cdot y$ is the intersection pairing $\int_{X} x \cup y$. The function $\Omega$ is needed to determine a line bundle on $\mathbb{T}$, a suitable section of which is the theta function. It is convenient to write $\Omega(x)=(-1)^{h(x)}$, where $h(x)$ is an integer-valued function that is defined modulo two.

In what follows, we will study the function $h(x)$ for the case that $X=S \times Y$, with $S$ a circle endowed with a spin structure of unbroken supersymmetry (that is, a non-bounding spin structure) and $Y$ an arbitrary nine-dimensional spin-manifold. We will find that if $x$ is an element of $H^{5}(Y ; \mathbb{Z})$, then

$$
h(x)=\int_{Y} \lambda \cup x
$$

where $\lambda$ is the integral characteristic class such that $2 \lambda=p_{1}(Y) . H^{5}(X ; \mathbb{Z})$ is generated by $H^{5}(Y ; \mathbb{Z})$ together with elements of the form $a \cup w$ with $a$ a generator of $H^{1}(S ; \mathbb{Z})=\mathbb{Z}$ and $w \in H^{4}(Y ; \mathbb{Z})$. So the function $\Omega(x)$ would be completely deter-

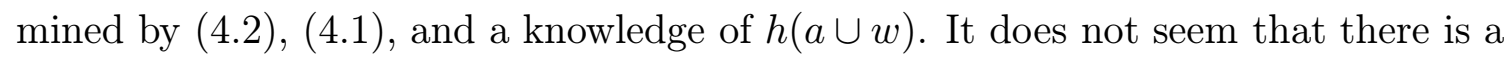

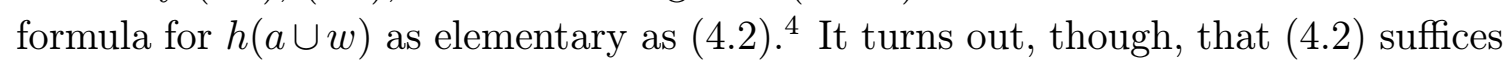
to answer the question raised in the introduction.

This comes about as follows. The use of the function $\Omega(x)$ to determine the partition function on a general $X$ is perhaps slightly esoteric. But this function has a more down-to-earth interpretation if $X$ is of the form $S \times Y$. In this case, the theory of the self-dual five-form $G_{5}$ on $X$ reduces on $Y$, at low energies, to a theory of a five-form on $Y$ that obeys no self-duality condition. We will call this field $G_{5}^{\prime}$. As $Y$ is nine-dimensional, the theory of $G_{5}^{\prime}$ is dual to a theory of a four-form field $G_{4}^{\prime}$ on $Y$. $G_{4}^{\prime}$ and $G_{5}^{\prime}$ are the curvatures of three-form and four-form potentials $C_{3}^{\prime}$ and $C_{4}^{\prime}$. The same theory on $Y$ can be described with either $C_{3}^{\prime}$ or $C_{4}^{\prime}$ as the dynamical variable.

\footnotetext{
${ }^{3}$ In general, $H^{5}(X ; \mathrm{U}(1))$ has components labeled by the torsion subgroup of $H^{6}(X ; \mathbb{Z})$; each component is a torus. This refinement will not be essential in our present discussion, and I suspect that the torsion can be fully taken into account only if one works with K-theory rather than cohomology, a task that we initiate in section 4.3 below. Note that in what follows, we write the product of differential forms as a wedge product, denoted $\wedge$, and the product of integral cohomology classes as a cup product, denoted $\cup$.

${ }^{4}$ For example, $(4.21)$ shows that $h(x)$ is independent of the spin structure for $x \in H^{5}(Y ; \mathbb{Z})$; but examples such as $Y=\widetilde{S} \times Y^{\prime}$ (with $\widetilde{S}$ another circle and $Y^{\prime}$ an eight-manifold) show that $h(a \cup w)$ does depend on the spin structure. In this case, for $w \in H^{4}\left(Y^{\prime} ; \mathbb{Z}\right), h(a \cup w)$ is equal to $\int_{Y^{\prime}} \lambda \cup w$ or 0 depending on whether one takes the supersymmetric or non-supersymmetric spin structure on $\widetilde{S}$. This can be seen by the methods of section
} 
$\Omega(x)$ has, as we have seen in section ${ }^{1} \overline{3}_{-1}$, the following straightforward interpretation when $X$ is of the form $S \times Y$ : it is a factor that must be included in the low energy path integral for the $G_{5}^{\prime}$ field on $Y$. To be more precise, if we regard the theory on $Y$ as the theory of a five-form $G_{5}^{\prime}$ with flux or characteristic class $x=\left[G_{5}^{\prime} / 2 \pi\right]$, then performing the path integral involves summing over $x$. The sum is weighted with a number of standard factors, such as an obvious factor coming from the kinetic energy of the $C_{4}^{\prime}$ field. In addition, we must include in the sum over $x$ the sign factor $\Omega(x)$, which according to ('A.

$$
\exp \left(i \pi \int_{Y} \lambda \cup x\right)=\exp \left(\frac{1}{2} i \int \lambda \wedge G_{5}\right) \text {. }
$$

On the other hand, if we represent the theory on $Y$ by a four-form $G_{4}^{\prime}$ with flux or characteristic class $u=\left[G_{4}^{\prime} / 2 \pi\right]$, then performing the path integral involves summing over $u$. The sign factor we must include in the path integral is in this case $\Omega(a \cup u)$ (since the relation between $G_{5}$ and $G_{4}^{\prime}$ is that the appropriate part of $G_{5}$ is $\left.a \wedge G_{4}\right)$, and we will not determine $\Omega(a \cup u)$. In addition to this in general unknown sign factor, the path integral over $G_{4}^{\prime}$ has another interesting effect, which arises by

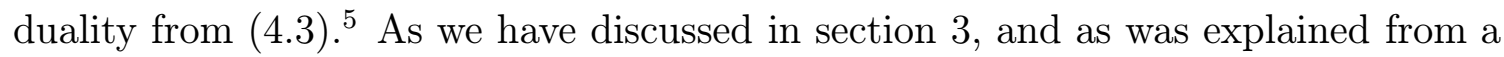
path integral point of view in [3] into a shift on the other side. In the present instance, since the phase in (' $\frac{1}{2} \lambda$ (times $G_{5}^{\prime}$ ), it is converted by duality into a shift in the periods of $G_{4}^{\prime}$ by that amount: for any four-cycle $U \in Y$,

$$
\int_{U} \frac{G_{4}^{\prime}}{2 \pi}=\frac{1}{2} \int_{U} \lambda \bmod \mathbb{Z}
$$

The last formula is essentially the result that we need. The problem posed in the introduction was to understand, starting with the quantum mechanics of the type-IIB self-dual five-form $G_{5}$, the fact that for any four-cycle $U$ in a type-IIA spacetime $X$,

$$
\int_{U} \frac{G_{4}}{2 \pi}=\frac{1}{2} \int_{U} \lambda \bmod \mathbb{Z}
$$

where here $G_{4}$ is the type-IIA four-form. If $X=S \times Y$ with $S$ a circle, then components of $\lambda$ with an index tangent to $S$ vanish topologically (since $\lambda$ is a pullback from $Y$ ), and the interesting case of ( $\left(\bar{A} . \bar{S}_{1}^{\prime}\right)$ is the case with $U$ a four-cycle in $Y$. Hence the interesting part of $G_{4}$ is the part with all indices tangent to $Y$. In the $T$-duality between type IIB and type IIA on $S \times Y$, this part of $G_{4}$ is related to the part of $G_{5}$ of the form $a \cup G_{4}$, with $a$ a generator of $H^{1}(S ; \mathbb{Z})$. So the relevant part of $G_{4}$ is the

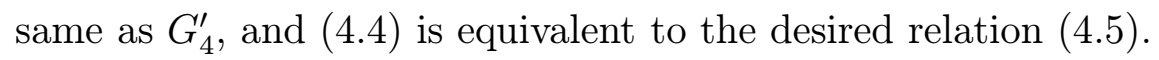

In the next subsection, we will justify the crucial formula ( $\left(\bar{A} .{ }_{2}^{\prime}\right)$. Then in section ' $\bar{A} . \overline{3}$, , we will propose a new description of $\Omega(x)$ in K-theory which may be more useful for understanding dualities and the role of torsion.

\footnotetext{
${ }^{5}$ And, conversely to what we are about to say, the undetermined sign factor $\Omega(a \cup u)$ in the $G_{4}^{\prime}$ theory will by duality, if it is not trivial, induce a shift in the periods of $G_{5}^{\prime}$.
} 


\subsection{Evaluation of $\Omega(x)$}

First we recall from [3]1] the definition of $\Omega(x)$ for a general $X$. We work on $Z=$ $S^{\prime} \times X$ where $S^{\prime}$ is a circle with a Neveu-Schwarz spin structure (that is, $S^{\prime}$ is a spin boundary). We fix a generator $a^{\prime}$ of $H^{1}\left(S^{\prime} ; \mathbb{Z}\right)=\mathbb{Z}$. For $x \in H^{5}(X ; \mathbb{Z})$, we set $z=a^{\prime} \cup x \in H^{6}\left(S^{\prime} \times X ; \mathbb{Z}\right)$. Now, if $W$ is any twelve-dimensional spin manifold with boundary $Z$ over which $z$ extends, ${ }^{6}$ we set

$$
h(x)=\int_{W} z \cup z
$$

and $\Omega(x)=(-1)^{h(x)}$. For $\Omega(x)$ to be well defined, $h(x)$ must be independent modulo 2 of the choice of $W$. This is so because for a closed twelve-dimensional spin manifold $W$ (that is, one whose boundary vanishes), $\int_{W} z \cup z$ is even for any $z \in H^{6}(W ; \mathbb{Z})$. (A proof of this assertion is cited in a footnote in [3-1; section 4].)

We could calculate more conveniently if we did not have to require $W$ to be a spin manifold. However, if we try to use the definition (' be spin, $h(x)$ would not be well-defined modulo 2 because $\int_{W} z \cup z$ is not necessarily even on a general twelve-manifold $W$. There is, however, an analogous quantity that is even in general; it is $\int_{W}(z \cup z+v \cup z)$, where $v$ is the (six-dimensional) Wu class of $W . v$ can be expressed in terms of Stieffel-Whitney classes; for our purposes, we can assume that $W$ is orientable, in which case the relation is $v=w_{2} \cup w_{4}$. Thus, we are tempted to generalize the definition of $h(x)$ to

$$
h(x)=\int_{W}\left(z \cup z+w_{2} \cup w_{4} \cup z\right)
$$

where $W$ is now required only to be oriented. (To make sense of the second integral, $z$ is reduced mod 2 and the integral is understood in terms of the cup product and integration in mod 2 cohomology.)

Some care is needed here. Though the right-hand side of ('A. the boundary of $W$ vanishes, some subtlety enters in defining the integral when $W$ has a non-zero boundary. An integral such as $\left(\bar{A}_{-} \bar{Z}_{1}\right)$ is not a topological invariant on a manifold with boundary unless the class that is being integrated is trivialized on the boundary; and even if it is, the integral depends on the choice of a trivialization on the boundary. (At the level of differential forms, this statement means that an integral $\int_{W} \Theta$, where $\Theta$ is a twelve-form, is not necessarily invariant under $\Theta \rightarrow \Theta+d \Lambda$ if $\Lambda$ is non vanishing on the boundary.) In the case of (' $\left.\bar{A}_{-} \overline{7}_{1}^{\prime}\right)$, if we understand $z$ near the boundary $Z$ of $W$ to be a pullback from $Z$, then $z \cup z$ vanishes near the boundary for dimensional reasons, and this trivialization is natural. We need more care with the term $w_{2} \cup w_{4} \cup z$.

\footnotetext{
${ }^{6}$ How to generalize the discussion if $W$ does not exist is discussed in $\left[3 i_{1}\right.$. We also give more general definitions of $\Omega(x)$ below and in section 'ta.3.
} 
As $z$ and $w_{4}$ are both in general non zero near the boundary, the only reason that $w_{2} \cup w_{4} \cup z$ vanishes near the boundary is that $w_{2}$ does, that is, the boundary manifold $Z$ is spin. A trivialization of $w_{2}$ near the boundary is a choice of spin structure on $Z$, and hence we will have to use the spin structure of $Z$ in defining the integral $\int_{W} w_{2} \cup w_{4} \cup z$ even though at first sight the integral appears not to depend on a choice of spin structure. A rather down-to-earth way to build in the spin structure of $Z$ is to restrict to the case that $W$ is a $\operatorname{Spin}^{c}$ manifold, with a $\operatorname{Spin}^{c}$ structure that extends the spin structure on $Z$. The $\operatorname{Spin}^{c}$ structure on $W$ determines an integral $\operatorname{lift}^{7} \alpha$ of $w_{2}(W)$ that is supported away from the boundary of $W$; to be more precise, it determines an element $\alpha$ of the relative cohomology group $H^{2}(W, \partial W ; \mathbb{Z})$ that reduces to $w_{2}(W) \bmod 2$. Moreover, if $W$ and $W^{\prime}$ are two $\operatorname{Spin}^{c}$ manifolds with (oppositely oriented) boundary $Z$ and $\operatorname{Spin}^{c}$ structures that extend the spin structure of $Z$, then upon gluing together $W$ and $W^{\prime}$ to make a closed twelvedimensional $\operatorname{Spin}^{c}$ manifold $\bar{W}$, the corresponding classes $\alpha$ and $\alpha^{\prime}$ glue together to an integral lift $\bar{\alpha}$ of $w_{2}(\bar{W})$. In fact, $\bar{\alpha}$ is derived from the $\operatorname{Spin}^{c}$ structure on $\bar{W}$ that is obtained by gluing those on $W$ and $W^{\prime}$. (Gluing $\alpha$ and $\alpha^{\prime}$ to make an integral lift $\bar{\alpha}$ of $w_{2}(\bar{W})$ would not work for arbitrary integral lifts $\alpha$ and $\alpha^{\prime}$ of $w_{2}(W)$ and $w_{2}\left(W^{\prime}\right)$; that is why it is important to derive $\alpha$ and $\alpha^{\prime}$ from $\operatorname{Spin}^{c}$ structures that extend that of $Z$.)

Finally, we get our more general definition of $h(x)$ :

$$
h(x)=\int_{W}\left(z \cup z+\alpha \cup w_{4} \cup z\right) .
$$

This is well-defined mod 2 because it is even for a closed twelve-dimensional $\operatorname{Spin}^{c}$ manifold $\bar{W}$.

Evaluation for $X=S \times Y$. We are ready to compute for the case that $X=$ $S \times Y$, with the supersymmetric (or non-bounding) spin structure on $S$. We have $Z=S^{\prime} \times X=S^{\prime} \times S \times Y$.

We want to evaluate $h(x)$ where $x$ is an element of $H^{5}(Y ; \mathbb{Z})$. We have $z=a^{\prime} \cup x$. To compute $h(x)$, we should write $Z$ as a boundary of a $\operatorname{Spin}^{c}$ manifold $W$ over which $z$ extends. We could try to take $W=D^{\prime} \times S \times Y$, where $D^{\prime}$ is a two-dimensional disc with boundary $S^{\prime}$. This is not convenient because $a^{\prime}$ does not extend over $D^{\prime}$. Instead, we let $D$ be a disc with boundary $S$, and set $W=S^{\prime} \times D \times Y$. The spin structure of $S$ does not extend over $D$ as a spin structure, but it extends as a $\operatorname{Spin}^{c}$ structure with

$$
\int_{D} \alpha=1
$$

\footnotetext{
${ }^{7}$ A $\operatorname{Spin}^{c}$ structure determines a $\operatorname{Spin}^{c}$ bundle that is informally $\mathcal{S} \otimes \mathcal{L}^{1 / 2}$ where $\mathcal{S}$ is the spin bundle and $\mathcal{L}$ is a line bundle with $c_{1}(\mathcal{L})$ congruent to $w_{2}(W) \bmod 2$. If $W$ is not spin, neither $\mathcal{S}$ nor $\mathcal{L}^{1 / 2}$ exists separately, but $\mathcal{S} \otimes \mathcal{L}^{1 / 2}$ and $\mathcal{L}$ do. $\alpha$ is defined as $c_{1}(\mathcal{L})$.
} 
As $z$ is a pullback from $S^{\prime} \times Y$, it extends over $W=S^{\prime} \times D \times Y$ as such a pullback. Now we can evaluate (' $\left(\bar{A} . \bar{z}_{1}^{\prime}\right)$. On dimensional grounds, since $z$ is pulled back from $S^{\prime} \times Y, z \cup z=0$. So we need only consider the integral over $S^{\prime} \times D \times Y$ of $\alpha \cup w_{4} \cup z=\alpha \cup w_{4} \cup a^{\prime} \cup x$. The integral is easily done because all factors are pullbacks from one of the factors in $S^{\prime} \times D \times Y\left(a^{\prime}\right.$ from $S^{\prime}, \alpha$ from $D$, and the others from $Y$ ). Using $\left({ }^{\prime} . \overline{9}_{-}^{\prime}\right)$ and $\int_{S^{\prime}} a^{\prime}=1$, we get

$$
h(x)=\int_{Y} w_{4} \cup x=\int_{Y} \lambda \cup x,
$$

where the two expressions are equivalent because on the spin manifold $Y, \lambda$ is an integral lift of $w_{4}$. This is the promised formula (' $\left.\bar{A}_{-} \overline{2}_{1}^{\prime}\right)$.

\subsection{K-Theory definition of $\Omega(x)$}

We have performed this computation in a framework [i] in which $\Omega(x)$ is defined as a function on middle-dimensional cohomology of type IIB. For two reasons, it seems that the definition should be reformulated in K-theory:

(1) In view of $T$-dualities which relate the Ramond-Ramond (RR) forms of different dimensions, and relate type IIB to type IIA, it seems unnatural to have a special formalism which only applies to the middle-dimensional $\mathrm{RR}$ form for type IIB, and does not apply at all for type IIA. If we define $\Omega(x)$ in K-theory, this will automatically include all of the RR forms of all even or all odd dimension, and may give a $T$-dual formalism.

(2) In view of what we now know about the RR fields, it seems unlikely that one can correctly take into account the torsion part of the RR fluxes without using K-theory instead of cohomology.

The rest of this section is devoted to an attempt to give a K-theory definition of $\Omega(x)$.

For type IIA at the level of differential forms, the total RR field $G=G_{0}+G_{2}+$ $G_{4}+\cdots$ is a sum of differential forms of all even orders. For type IIB, one has instead a sum $G=G_{1}+G_{3}+\cdots$ of differential forms of all odd orders. In passing to K-theory, we will assume that for type IIA, the RR flux should be regarded as an element $x \in K(X)$. For type IIB, it should be regarded as an element $x \in K^{1}(X)$.

We will first define a $\mathbb{Z}_{2}$-valued function $\Omega(x)=(-1)^{h(x)}$ for $x \in K(x)$, that is, for type IIA. We want

$$
\Omega(x+y)=\Omega(x) \Omega(y)(-1)^{(x, y)},
$$

where $(x, y)$ should be an integer-valued bilinear form on $K(X)$ that generalizes the intersection pairing on cohomology. Moreover, we want $(x, y)=-(y, x)$, so that $\Omega(x)$ can be used to define a line bundle on a torus $K(X ; \mathbb{R} / \mathbb{Z}) / K(X ; \mathbb{Z})$ (by analogy with 
what is done for the middle-dimensional cohomology in [i] is given by index theory. For any $w \in K(x)$, let

$$
i(w)=\int_{X} \widehat{A}(X) \operatorname{ch}(w)
$$

be the index of the Dirac operator with values in $w$. In ten-dimensions, the only terms in $\operatorname{ch}(w)$ that contribute are terms of degree $4 k+2$ for some integer $k$. These terms are odd under $w \rightarrow \bar{w}$ (complex conjugation of the bundle) so

$$
i(w)=-i(\bar{w})
$$

Then we set

$$
(x, y)=i(x \otimes \bar{y})
$$

which obeys $(x, y)=-(y, x)$ by virtue of (14.13is ). This pairing vanishes if $x$ or $y$ is torsion; it can be proved that on $K(X) \bmod$ torsion, it is unimodular.

There is one more thing we should know about index theory in ten dimensions. If $w$ is a real bundle, then $i(w)=0$ because of (' $\left.\bar{A} . \overline{1} \overline{3}_{1}^{\prime}\right)$. But there is nonetheless a natural invariant of $w$ that can be defined using index theory. This is the "mod 2 index," the number of positive chirality zero modes of the Dirac operator with values

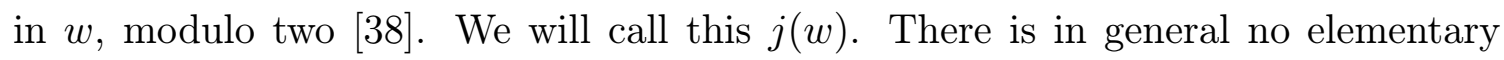
formula for $j(w)$. But if the complexification of $w$ is of the form $x \oplus \bar{x}$ for some complex bundle $x$, then

$$
j(w)=i(x) \bmod 2 .
$$

In fact, $i(x)=n_{+}(x)-n_{-}(x)$, where $n_{+}(x)$ and $n_{-}(x)$ are respectively the number of positive and negative chirality zero modes with values in $x$. Since in ten dimensions, complex conjugation reverses the chirality, we have $n_{-}(x)=n_{+}(\bar{x})$, so modulo 2 we have $i(x)=n_{+}(x)+n_{+}(\bar{x})=n_{+}(w)=j(w)$.

We now can define $h(x)$, and hence $\Omega(x)$, for type IIA. We simply set

$$
h(x)=j(x \otimes \bar{x}) .
$$

We must verify (' $\left.\overline{4} . \overline{1} \overline{1}_{1}^{\prime}\right)$. If $z=x \oplus y$, then $z \otimes \bar{z}=x \otimes \bar{x} \oplus y \otimes \bar{y} \oplus w$, with $w=x \otimes \bar{y} \oplus y \otimes \bar{x}$. So

$$
\begin{aligned}
h(x+y) & =j(z \otimes \bar{z})=j(x \otimes \bar{x})+j(y \otimes \bar{y})+j(w)= \\
& =h(x)+h(y)+i(x \otimes \bar{y})=h(x)+h(y)+(x, y)
\end{aligned}
$$

as required.

We also want the analogous definition for type IIB. In this case, we want to define a suitable function $\Omega(x)$ for $x \in K^{1}(X)$. We interpret $K^{1}(X)$ as $\widetilde{K}\left(X \times \mathbf{S}^{1}\right)$, 
the subset of $K\left(X \times \mathbf{S}^{1}\right)$ consisting of elements that are trivial if restricted to $X$. For $x, y \in K^{1}(X)$, we have $x \otimes \bar{y} \in K^{2}(X)=\widetilde{K}\left(X \times \mathbf{S}^{1} \times \mathbf{S}^{1}\right)$, and we define

$$
(x, y)=\int_{X \times \mathbf{S}^{1} \times \mathbf{S}^{1}} \widehat{A}\left(X \times \mathbf{S}^{1} \times \mathbf{S}^{1}\right) \operatorname{ch}(x \otimes \bar{y}) .
$$

This integer-valued function again obeys $(x, y)=-(y, x)$.

Now we want to define $\Omega(x)$. Here there is a slight subtlety. The element $x \otimes \bar{x}$ of $\widetilde{K}\left(X \times \mathbf{S}^{1} \times \mathbf{S}^{1}\right)$ is replaced by its complex conjugate if one exchanges the two $\mathbf{S}^{1}$ 's. In addition, it is trivial if restricted to $X \times \mathbf{S}^{1} \times p$ or $X \times p \times \mathbf{S}^{1}$, with $p$ a point in one of the $\mathbf{S}^{1}$ 's. These properties ensure that $x \otimes \bar{x}$ can be interpreted as an element of $K R\left(X \times \mathbf{S}^{2}\right)$, where the real involution used in defining $K R$ is a reflection of one coordinate of $\mathbf{S}^{2}$. (By collapsing $\mathbf{S}^{1} \times p$ and $p \times \mathbf{S}^{1}$, one maps $\mathbf{S}^{1} \times \mathbf{S}^{1}$ to $\mathbf{S}^{2}$; the map that exchanges the two factors of $\mathbf{S}^{1} \times \mathbf{S}^{1}$ becomes a reflection of one coordinate in $\mathbf{S}^{2}$.) By the periodicity theorem of $K R$ theory [$\mathrm{KO}(X)$. So $x \otimes \bar{x}$ maps to an element $w \in \mathrm{KO}(X)$, and we define $h(x)=j(w)$. The proof of $\left(' \bar{A} . \overline{1} \overline{1}_{1}^{\prime}\right)$ is rather as before.

\section{Systematic analysis for M5-brane}

In this section, we will carry out an analysis of the other problem mentioned in the introduction - the relation of the M5-brane to the D4-brane - analogous to what we have seen in section 4 for type IIA/IIB. The discussion will proceed in the following stages: first we will summarize results; then we will compute by hand; then we will place the computation more systematically in the framework of [i]

\subsection{Outline}

Let $V$ be the worldvolume of an M5-brane in an M-theory spacetime $M$. In general, $V$ is oriented, but perhaps not spin.

The subtle part of the quantum mechanics of the M5-brane is to quantize the chiral two-form, which has a characteristic class $x \in H^{3}(V ; \mathbb{Z})$. The general framework for doing so is analogous to what we summarized in the last section. Roughly speaking, one defines a $\mathbb{Z}_{2}$-valued function $\Omega(x)=(-1)^{h(x)}$ on $H^{3}(V ; \mathbb{Z})$, obeying the usual relation

$$
\Omega(x+y)=\Omega(x) \Omega(y)(-1)^{(x, y)} .
$$

This enables one to construct a theta function that determines the partition function of the chiral two-form. ${ }^{8}$

In general, there is no elementary formula for $\Omega(x)$. However, for the case that the M5-brane can be related to a D4-brane, there is such a formula, in part. This is the case that $V=S \times R$, with $S$ a circle with supersymmetric spin structure and $R$

\footnotetext{
${ }^{8}$ This description omits a twist that we recall in section 5.2
} 
a five-manifold. In this case, we will justify the following assertion about $\Omega(x)$ : if $x$ is an element of $H^{3}(R ; \mathbb{Z})$, then

$$
h(x)=\int_{R} w_{2}(R) \cup x .
$$

Here to make sense of this integral, $x$ should be reduced mod 2, and the integral is understood as an intersection number in mod 2 cohomology. To fully determine $\Omega(x)$ (with the help of $\left(\underline{w_{i}} \overline{1}\right)$ ), we would also need to compute $\Omega(a \cup w)$ for $a$ a generator of $H^{1}(S ; \mathbb{Z})$ and $w \in H^{2}(R ; \mathbb{Z})$. It does not seem that there is a formula for $\Omega(a \cup w)$ as elementary as $(\underline{6} \cdot \overline{2} . \overline{2})$.

In general, the physical application of $\Omega(x)$ is rather subtle. But (as in the case we considered in section 'A- $\bar{l}_{-}$), the interpretation of $\Omega(x)$ is more straightforward when $V=S \times R$. In this case, the chiral two-form on $V$ reduces on $R$ to an ordinary two-form field $B_{2}$ with field strength $T_{3}=d B_{2}$ and characteristic class $x=\left[T_{3} / 2 \pi\right]$, or (by duality) to a one-form field $B_{1}$ with two-form field strength $T_{2}=d B_{1}$ and characteristic class $v=\left[T_{2} / 2 \pi\right]$. In the description by a two-form field, the evaluation of the path integral includes a summation over $x$ in which one must include the sign factor $\Omega(x)$. This factor can be understood as coming from a term in the lagrangian

$$
i \pi \int_{R} w_{2} \cup x
$$

In the dual description by a one-form field, the evaluation of the path integral includes a summation over $v$. In evaluating this sum, one includes a sign factor $\Omega(a \cup v)$ for which we will not obtain an explicit general formula. In addition (as in

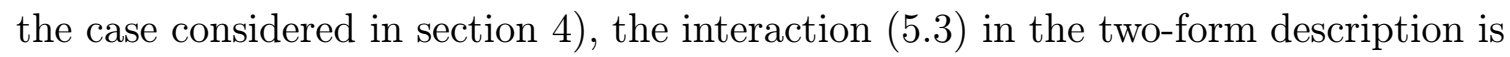
dual in the one-form description to a shift in the periods of $T_{2}$. The dual of (15.3is a shifted quantization law,

$$
\int_{U} \frac{T_{2}}{2 \pi}=\frac{1}{2} \int_{U} w_{2} \bmod \mathbb{Z} .
$$

The shift means that $B_{1}$, whose curvature is $T_{2}$, is not a " $\mathrm{U}(1)$ gauge field," but rather defines a $\operatorname{Spin}^{c}$ structure on $R$. (Reciprocally, the sign factor $\Omega(a \cup v)$ will in general determine a shift in the periods of $T_{3}$.)

Since $R$ might not be $\operatorname{Spin}^{c}$, something is missing in the discussion so far. There is an important difference between ( $(2.2)$ and the analogous formula $h(x)=\int_{R} \lambda \cup x$ that we met in section 我. As $\lambda$ is an integral cohomology class, the integral $\int_{R} \lambda \cup x$ vanishes if $x$ is torsion; that is why torsion was not very important in section However, $w_{2}$ is a $\mathbb{Z}_{2}$-valued cohomology class, and $\int w_{2} \cup x$ can perfectly well be non zero for torsion $x$. We will show momentarily that precisely when $R$ is not $\operatorname{Spin}^{c}$, there is a torsion class $x_{0}$ with $\Omega\left(x_{0}\right)=-1$. It follows (since $\left(x, x_{0}\right)=0$ for all $x$, given that $x_{0}$ is torsion) that $\Omega\left(x+x_{0}\right)=-\Omega(x)$ for all $x$. In determining the partition 
function of the M5-brane, the factor $\Omega(x)$ is the only factor that is not invariant under $x \rightarrow x+x_{0}$. (For example, since $x_{0}$ is torsion, the ordinary kinetic energy of the two-form field does not receive a contribution from $x_{0}$.) The contributions to the partition function from $x$ and $x+x_{0}$ will therefore cancel in pairs, and the partition function of the M5-brane vanishes. This vanishing cannot be lifted by inserting local operators (which do not detect a flat two-form field with characteristic class $x_{0}$ ), and so should be understood as a sort of global anomaly. Existence of this anomaly gives an M5-brane explanation of the fact that in type IIA, the D4-brane world-volume should be $\operatorname{Spin}^{c}$.

The existence of $x_{0}$ when $R$ is not $\operatorname{Spin}^{c}$ follows from some basic facts in algebraic topology. The cup product gives a map

$$
H^{2}(R ; \mathrm{U}(1)) \times H^{3}(R ; \mathbb{Z}) \longrightarrow H^{5}(R ; \mathrm{U}(1))=\mathrm{U}(1)
$$

which by Poincaré and Pontryagin duality is a perfect pairing. The "perfectness" means that every homomorphism $H^{3}(R ; \mathbb{Z}) \rightarrow \mathrm{U}(1)$ is $x \rightarrow \int_{R} \theta \cup x$ for some $\theta \in$ $H^{2}(R ; \mathrm{U}(1))$, and every homomorphism $H^{2}(R ; \mathrm{U}(1)) \rightarrow \mathrm{U}(1)$ is $\theta \rightarrow \int_{R} \theta \cup x$ for some $x \in H^{3}(R ; \mathbb{Z})$. If one restricts the pairing in $\left({ }_{1} .5 .51\right)$ to the torsion subgroup $H_{\text {tors }}^{3}(R ; \mathrm{U}(1))$, then one gets an analogous perfect pairing

$$
\bar{H}^{2}(R ; \mathrm{U}(1)) \times H_{\text {tors }}^{3}(R ; \mathbb{Z}) \longrightarrow \mathrm{U}(1) .
$$

Here $\bar{H}^{2}(R ; \mathrm{U}(1))$ is the group of components of $H^{2}(R ; \mathrm{U}(1))$ (in other words, it is the quotient of $H^{2}(R ; \mathrm{U}(1))$ by the connected component containing the identity). The formula $h=\int_{R} w_{2} \cup x_{0}$ is equivalent to $\Omega=\int_{R} i\left(w_{2}\right) \cup x_{0}$ where $i: \mathbb{Z}_{2} \rightarrow \mathrm{U}(1)$ is the embedding of $\mathbb{Z}_{2}$ into $U(1)$. So perfectness of $\left({ }^{\prime}-\overline{6}_{1}^{\prime}\right)$ means that a torsion class $x_{0}$ with $\Omega\left(x_{0}\right)=-1$ exists precisely if $i\left(w_{2}\right)$ is not in the identity component of $H^{2}(R ; \mathrm{U}(1))$. Now consider the commutative diagram

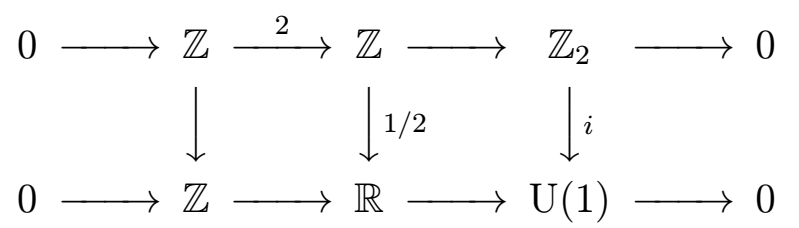

where the first horizontal map in the top row is multiplication by 2 , the other horizontal maps are obvious inclusions and reductions, the first vertical map is the identity, the second vertical line is multiplication by $1 / 2$, and the last is $i$. Let $\beta: H^{2}\left(R ; \mathbb{Z}_{2}\right) \rightarrow H^{3}(R ; \mathbb{Z})$ be the Bockstein derived from the first row, and let $\beta^{\prime}: H^{2}(R ; \mathrm{U}(1)) \rightarrow H^{3}(R ; \mathbb{Z})$ be the Bockstein derived from the second. The condition that $R$ is not $\operatorname{Spin}^{c}$ is $\beta\left(w_{2}\right) \neq 0$; in fact, $W_{3}(R)=\beta\left(w_{2}\right)$ is the obstruction to $\operatorname{Spin}^{c}$ structure. The condition that $i\left(w_{2}\right)$ not be in the identity component of $H^{2}(R ; \mathrm{U}(1))$ is that $\beta^{\prime}\left(i\left(w_{2}\right)\right) \neq 0$. Commutativity of the above diagram implies that $\beta^{\prime}=i \beta$. So $i\left(w_{2}\right)$ is not in the identity component, and a torsion $x_{0}$ with $\Omega\left(x_{0}\right)=-1$ exists, if and only if $W_{3}(R) \neq 0$ and $R$ is not $\operatorname{Spin}^{c}$. 
Generalizations. This discussion of a global anomaly is not limited to the case that $V=S \times R$. More generally, the M5-brane is anomalous whenever there is a torsion class $x_{0}$ with $\Omega\left(x_{0}\right)=-1$. However, it is hard in general to give a criterion for existence of $x_{0}$.

I will now briefly suggest how these anomalies can be removed by turning on background fields. In the discussion so far, we have taken the Neveu-Schwarz threeform field $H$ of type IIA, and the corresponding M-theory four-form field $G$, to be topologically trivial. Naively, the classical equations $d T_{2}=H$ and $d T=G$ (where $T_{2}$ is the two-form on a D4-brane and $T$ is the self-dual three-form on an M5-brane) imply that $H$ and $G$ should be trivial when restricted to the D4- and M5-brane worldvolumes. However, taking into account the global anomalies, the general statement for type IIA is [i], ', 'în]

$$
\left.H\right|_{R}=W_{3}(R)
$$

where $\left.H\right|_{R}$ is shorthand for the restriction to $R$ of the characteristic class of $H$. The analog of this condition for the M5-brane should apparently be the following. Under the perfect pairing

$$
\bar{H}^{3}(V ; \mathrm{U}(1)) \times H_{\text {tors }}^{3}(V ; \mathbb{Z}) \longrightarrow \mathrm{U}(1)
$$

analogous to the one considered above, the function $x_{0} \rightarrow \Omega\left(x_{0}\right)$ (for $x_{0}$ torsion) corresponds to an element $\theta \in \bar{H}^{3}(V ; \mathrm{U}(1))$. The general statement about the restriction of $G$ to $V$ should apparently be

$$
\left.G\right|_{V}=\beta^{\prime}(\theta),
$$

where as above $\beta^{\prime}$ is the Bockstein. This reduces to (15.8. and I suspect that it holds in general.

\subsection{Direct computation}

Let us next attempt to directly imitate the computation in section '重. To begin with, we assume that $V$ is spin.

For $x \in H^{3}(V ; \mathbb{Z})$, we want to define a suitable $\mathbb{Z}_{2}$-valued function $\Omega(x)=$ $(-1)^{h(x)}$. We let $Z=S^{\prime} \times V$ (with $S^{\prime}$ a circle) and set $z=a^{\prime} \cup x$ with $a^{\prime}$ a generator of $H^{1}\left(S^{\prime} ; \mathbb{Z}\right) .{ }^{9}$ Then, assuming that $Z$ is the boundary of an eight-dimensional spin manifold $W$ over which $z$ extends, one is tempted to set $h(x)=\int_{W} z \cup z$. This is not well-defined modulo 2, because in general for a closed eight-dimensional spin

\footnotetext{
${ }^{9}$ The following computation has a very similar structure to the one in section $\overline{4} \overline{4}_{1}^{\prime}$ although a few details are different. To try to bring out the analogy, and hopefully without causing confusion, we will use some of the notation of section $\overline{4}_{1}^{4}$ for objects that play the analogous role here. The sevenmanifold $Z$ is analogous to the eleven-manifold called $Z$ in section 'inis; likewise, the eight-manifold $W$ of boundary $Z$ will be analogous to the twelve-manifold called $W$ in section use the names $S^{\prime}, a^{\prime}, x$, and $z$ for objects that play an analogous role to objects of the same name in section
} 
manifold $W, \int_{W} z \cup z$ is not even. The quantity which is always even for a closed eight-dimensional spin manifold with a given $z \in H^{4}(W ; \mathbb{Z})$ is $\int_{W}(z \cup z+\lambda \cup z)$ (where $\lambda$ is the integral characteristic class with $2 \lambda=p_{1}(W)$ ), so we set

$$
h(x)=\int_{W}(z \cup z+\lambda \cup z) .
$$

Here we need, as in the analogous discussion in section 'A, to make sense of the integral $\int_{W} \lambda \cup z$ on the manifold-with-boundary $W$. This integral needs some explanation, because in general neither $\lambda$ nor $z$ vanishes on the boundary of $W$. The approach taken in [3] we would use the function $\Omega(x)$ to quantize the torus $\mathbb{T}=H^{3}(V ; \mathbb{R}) / H^{3}(V ; \mathbb{Z})$ that parametrizes flat three-form fields $C$ on $V \bmod$ gauge transformations. The $\lambda \cup z$ term in $\left(15.1 \overline{1}_{1}^{\prime}\right)$ means that the torus that we can naturally quantize is not $\mathbb{T}$ but the torus $\mathbb{T}^{\prime}$ that parametrizes, up to gauge transformations, $C$-fields of curvature $\lambda / 2$. ( $\mathbb{T}$ is isomorphic to $\mathbb{T}^{\prime}$, by the map $C \rightarrow C+C_{0}$ where $C_{0}$ is any $C$-field of curvature $\lambda / 2$, but there is no canonical isomorphism between $\mathbb{T}$ and $\mathbb{T}^{\prime}$.) A heuristic way to explain the shift from $\mathbb{T}$ to $\mathbb{T}^{\prime}$ is that $z \rightarrow z-\lambda / 2$ eliminates the $z \cup \lambda$ term in (6.1.1); for more information, see [3in in ('5.1 $\left.\overline{1}_{1}^{1}\right)$ (described to me by M. Hopkins and I.M. Singer) is as follows. The $\lambda$ class of a seven-dimensional spin manifold such as $Z$ is always even. ${ }^{10}$ Since we only want to define $h(x)$ modulo 2 , we can interpret the integral $\int_{W} \lambda \cup z$ as an integral in mod 2 cohomology, replacing $\lambda$ and $z$ by their mod 2 reductions $\bar{\lambda}$ and $\bar{z}$. Since $\bar{\lambda}$ vanishes when restricted to the boundary of $W$, we can pick a trivialization of it; once such a trivialization is picked, the integral $\int_{W} \bar{\lambda} \cup z$ makes sense. The relation between the two approaches is that a trivialization of $\lambda \bmod 2$ gives a way of identifying $\mathbb{T}$ and $\mathbb{T}^{\prime}$.

The details in the last paragraph will not play a major role in the present paper. The reason is that, with $V=S \times R$, we will compute $\Omega(x)$ only for $x \in H^{3}(R ; \mathbb{Z})$. This means that on $Z=S^{\prime} \times V=S^{\prime} \times S \times R$, both $\lambda$ and $z=a^{\prime} \cup x$ are pullbacks from $S^{\prime} \times R$. In trivializing $\lambda \bmod 2$ on $Z$, we can restrict ourselves to consider only trivializations that are pulled back from $R$, and the choice of such a trivialization does not affect the integral $\int_{W} \lambda \cup z$. At the level of differential forms, this last statement means that under $\lambda \rightarrow \lambda+d \gamma, \int_{W} \lambda \cup z$ changes by $\int_{S^{\prime} \times S \times R} \gamma \cup z$, which vanishes for $\gamma$ and $z$ both being pullbacks from $S^{\prime} \times R$. Hence there is a completely canonical $\Omega(x)$ for the $x$ we will consider, and this is what we will evaluate.

Just as in section 'A. ${ }^{2}$, , it is inconvenient to calculate with $W$ required to be a spin manifold. We can readily generalize the discussion to permit $V$ and $W$ to be Spin $^{c}$ manifolds, not necessarily spin, as follows. A Spin ${ }^{c}$ manifold $W$ (with a chosen

\footnotetext{
${ }^{10}$ The intersection form of the eight-manifold $B=\mathbf{S}^{1} \times V$ is even, so the relation $\int_{B}(x \cup x+\lambda \cup x) \cong$ 0 modulo 2 for all $x \in H^{4}(B ; \mathbb{Z})$ reduces to $\int_{B} \lambda \cup x \cong 0$ modulo 2 for all $x$. This implies that $\lambda$ is divisible by 2 .
} 
Spin $^{c}$ structure) has a two-dimensional class $\alpha \in H^{2}(W ; \mathbb{Z})$, which reduces mod 2 to $w_{2}(W)$. In addition, on such a manifold $p_{1}-\alpha^{2}$ is divisible by 2 , and there is an integral characteristic class $\lambda$ such that ${ }^{11} 2 \lambda=p_{1}-\alpha^{2}$. Moreover, for any $x \in H^{4}(W ; \mathbb{Z}), \int_{W}(x \cup x+\lambda \cup x)$ is always even. ${ }^{12}$ So we can evaluate (15.1 $\left.11_{1}^{1}\right)$ for any Spin $^{c}$ manifold $W$, with $\lambda$ as just defined.

We will now consider $h(x)$ for $V=S \times R$. We assume first that $R$ is $\operatorname{Spin}^{c}$. We give $V$ a $\operatorname{Spin}^{c}$ structure that is the product of the supersymmetric (or unbounding) spin structure on $S$ with the given $\operatorname{Spin}^{c}$ structure on $R$. We set $Z=S^{\prime} \times V=$ $S^{\prime} \times S \times R$. Suppose that $x \in H^{3}(R ; \mathbb{Z})$. Then as in section ' $1 \overline{4} .21, Z$ is the boundary of a $\operatorname{Spin}^{c}$ manifold $W=S^{\prime} \times D \times R$, where $D$ is a disc of boundary $S$; and $z=a^{\prime} \cup x$ extends over $W$ as a pullback from $S^{\prime} \times R$. The $\operatorname{Spin}^{c}$ structure on $W$ is the product of a spin structure on $S^{\prime}$, the given $\operatorname{Spin}^{c}$ structure on $R$ with two-dimensional class $\alpha_{R}$, and a $\operatorname{Spin}^{c}$ structure on $D$ with a two-dimensional class $\alpha_{D}$ such that $\int_{D} \alpha_{D}=1$. (The reason for the last statement is the same as in section 'A. $\bar{A}_{-}$: the supersymmetric spin structure on $S$ does not extend over $D$ as a spin structure, but it extends as a Spin $^{c}$ structure with $\int_{D} \alpha_{D}=1$.) We have $p_{1}(W)=p_{1}(R)$ and $\alpha(W)=\alpha_{D}+\alpha_{R}$; also, $\alpha_{D} \cup \alpha_{D}=0$ since $D$ is two-dimensional. We can compute the $\lambda$ class of $W$ : $\lambda(W)=\left(p_{1}(W)-\alpha(W)^{2}\right) / 2=\lambda(R)-\alpha_{D} \cup \alpha_{R}$. It follows that

$$
h(x)=\int_{W}(z \cup z+\lambda(w) \cup z)=\int_{W}\left(z \cup z+\lambda(R) \cup z-\alpha_{D} \cup \alpha_{R} \cup z\right) .
$$

On the right-hand side, only the term $\alpha_{D} \cup \alpha_{R} \cup z$ contributes to the integral, as the others are pullbacks from $S^{\prime} \times R$. Using $z=a^{\prime} \cup x$, with $\int_{S^{\prime}} a^{\prime}=1$, and $\int_{D} \alpha_{D}=1$, we get

$$
h(x)=-\int_{R} \alpha_{R} \cup x .
$$

Since $\alpha_{R}$ is congruent to $w_{2}(R) \bmod 2$, this is equivalent to the promised formula

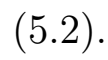

\footnotetext{
${ }^{11}$ More generally, any real oriented vector bundle $E$ with $w_{2}(E)=0$ has an integral characteristic class $\lambda$ with $2 \lambda(E)=p_{1}(E)$. If $W$ is $\operatorname{Spin}^{c}$, let $J$ be a real two-plane bundle with Euler class $\alpha$, and let $E=T W \oplus J$ (with $T W$ being the tangent bundle to $W$ ). Then $w_{2}(E)=0$, and $\lambda(E)$ is the desired class with $2 \lambda=p_{1}(E)=p_{1}(T W)-\alpha^{2}$.

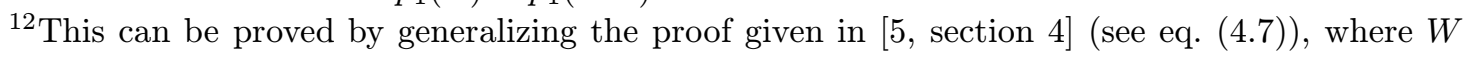
was assumed to be spin. Let $J$ be a real two-plane bundle over $W$ with Euler class $\alpha$, and let $N$ be the direct sum of $J$ with a trivial rank three bundle. Let $K$ be a twelve-manifold that is the unit sphere bundle in $N ; K$ is spin. Let $\pi: K \rightarrow W$ be the projection, let $x$ be any element of $H^{4}(W ; \mathbb{Z})$, and let $u$ be an element of $H^{4}(K ; \mathbb{Z})$ with $\pi_{*}(u)=1$ and $u \cup u=0$. (Such a $u$ can be constructed as the Poincaré dual of a section of $\pi$.) Consider, as in [iñ with characteristic class $u+\pi^{*}(x)$. If $i(B)$ is the index of the Dirac operator on $K$ with values in $B$ (in the adjoint representation), then $i(B)$ is even (because $B$ is real and $K$ has dimension of the

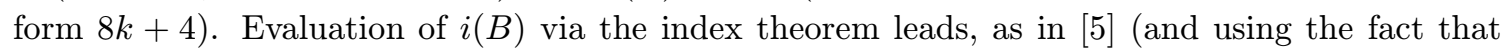
$\lambda(K)=\pi^{*}(\lambda(W))$ where $\lambda(W)$ is defined as in the last footnote using the $\operatorname{Spin}^{c}$ structure of $\left.W\right)$, to $i(B)=\int_{W}(x \cup x+\lambda(W) \cup x)$, and so this expression is even.
} 
So far we have assumed that $V$ is $\operatorname{Spin}^{c}$. Otherwise, the $\lambda$ class is no longer available, but we still have the Wu class $v$ in $\bmod 2$ cohomology, with $\int_{W}(x \cup x+v \cup x)$ even. In eight dimensions,

$$
v=w_{2}^{2}+w_{4}
$$

So the definition of $h(x)$ should be

$$
h(x)=\int_{W}\left(z \cup z+\left(w_{4}+w_{2}^{2}\right) \cup z\right) .
$$

Here we have given the most natural topological definition. In section "5.5. verify that it is equivalent to the physics-based definition in [3] $\left.\overline{3}_{1}\right]$.

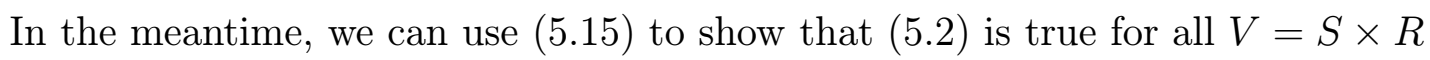
and $x \in H^{3}(R ; \mathbb{Z})$, whether or not $R$ is $\operatorname{Spin}^{c}$. For this, we note that it follows from ( $(5.14)$ is that if $R$ is the boundary of an oriented manifold $\widetilde{R}$ over which $x$ extends, then $h(x)=0$. For in this case, we can set $W=S^{\prime} \times S \times \widetilde{R}$, and the integral defining $h(x)$ vanishes as $x, w_{2}$, and $w_{4}$ are all pullbacks from $S^{\prime} \times \widetilde{R}$. This bordism property can be used to reduce to the case that $R$ is $\operatorname{Spin}^{c}$. Indeed, we can always find an oriented six-manifold $\widetilde{R}$ whose boundary is $R-R_{1}-R_{2}$ (the minus signs keep track of the orientations), where $x$ extends over $\widetilde{R}$ and vanishes on $R_{2}$, and $R_{1}$ is $\operatorname{Spin}^{c}{ }^{13}$ The bordism property implies that $h(x)$ is the same whether computed on $S \times R$ or $S \times R_{1}\left(R_{2}\right.$ does not contribute as $x$ vanishes on $\left.R_{2}\right)$. As $R_{1}$ is $\operatorname{Spin}^{c}$, we can use our previous result: $h(x)=\int_{R_{1}} w_{2} \cup x$. Since the characteristic class $w_{2}(R)$ automatically extends over $\widetilde{R}$, one has $\int_{R} w_{2} \cup x=\int_{R_{1}} w_{2} \cup x$. Hence $h(x)=\int_{R} w_{2} \cup x$ whether or not $R$ is $\operatorname{Spin}^{c}$.

We could have made a much more extensive use of bordism in the present paper. Indeed, we could have used the fact that $\Omega_{5}^{\operatorname{Spin}^{c}}(K(\mathbf{Z}, 2))=\mathbb{Z}$, generated by $\int \alpha \cup x$, to show that (15) is the only non-zero bordism-invariant formula for $h(x)$ in the $\operatorname{Spin}^{c}$ case, whereupon we could deduce from the example considered in section 120 that $(15 . \overline{2})$ is correct. We similarly could use the fact that $\widetilde{\Omega}_{9}^{\mathrm{Spin}}(K(\mathbf{Z}, 5))=\mathbb{Z}$, generated by $\int \lambda \cup x$, plus invariance under bordism, to reduce the computation in section ' $1 \bar{A} .21$ to the special case considered in section $2_{i}$. This would give short cuts to the desired results, but we have chosen instead to base our computations on a better understanding of

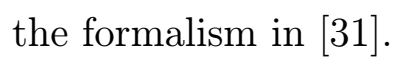

\footnotetext{
${ }^{13}$ The precise mathematical statement here is that $\Omega_{5}(K(\mathbb{Z}, 3))$, the bordism group of oriented five-manifolds equipped with a three-dimensional cohomology class $x$, is $\mathbb{Z}_{2} \times \mathbb{Z}_{2}$, a complete set of invariants being $\int w_{2} \cup x$ and $\int w_{2} \cup w_{3}$. (This statement and analogous ones cited in the next paragraph were provided by R. Stong, along with proofs.) So for the bordism group, we can pick two generators $R_{1}^{\prime}$ and $R_{2}^{\prime}$, where $\int w_{2} \cup x$ is non zero on $R_{1}^{\prime}$ and zero on $R_{2}^{\prime}$, and $\int w_{2} \cup w_{3}$ is non zero on $R_{2}^{\prime}$ and zero on $R_{1}^{\prime}$. Moreover, one can pick $R_{1}^{\prime}$ to be $\operatorname{Spin}^{c}$, and one can assume that $x$ vanishes on $R_{2}^{\prime}$. The fact that $R_{1}^{\prime}$ and $R_{2}^{\prime}$ generate the bordism group means that $R-R_{1}-R_{2}$ is a boundary, where the $R_{i}$ are as in the text and each $R_{i}$ is equal to $R_{i}^{\prime}$ or empty, depending on the values of the invariants of $R$.
} 


\subsection{Comparison to physical definition}

It remains to compare the obvious topological definition (15.

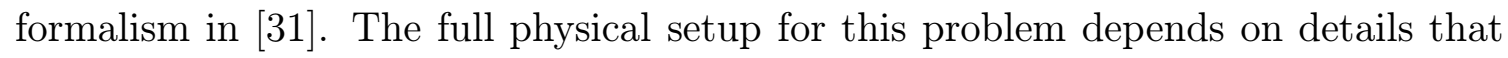
we have so far omitted. The M5-brane worldvolume $V$ is embedded in an elevenmanifold $M . V$ is orientable (but not necessarily spin), and $M$ is spin. Let $N$ be the normal bundle to $V$ in $M$. The condition for $M$ to be spin is

$$
w_{1}(N)=0, \quad w_{2}(N)=w_{2}(V) .
$$

Also, the Euler class of $N$ vanishes (or equivalently, as $N$ is of odd rank, $w_{5}(N)=0$ ), for reasons explained in $\left[{ }_{1}^{5}\right.$, section 5]. Another part of the data is the four-form field $G$ of M-theory. It is of the form

$$
\frac{G}{2 \pi}=\frac{\lambda(M)}{2}+g
$$

where $g$ is an integral class. Moreover, if $U$ is a small four-sphere linking $V$ in $M$, then

$$
\int_{U} g=1
$$

since the fivebrane has unit charge.

Let $P$ be the submanifold of $M$ consisting of all points a distance $\epsilon$ from $V$, for some very small $\epsilon . P$ is a four-sphere bundle over $V$. Let $\pi: P \rightarrow V$ be the

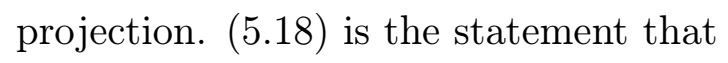

$$
\pi_{*}(g)=1
$$

This uniquely determines $g$ modulo $g \rightarrow g+\pi^{*}(y)$ for $y \in H^{4}(V ; \mathbb{Z})$. Note that $\pi_{*}(g \cup g)$ is invariant mod 2 under such a transformation of $g$. Hence, its $\bmod 2$ reduction does not depend on the choice of $g$. In fact,

$$
\pi_{*}(g \cup g) \cong w_{4}(N) \bmod 2
$$

To prove this, since the left-hand side is independent of the choice of $g$ modulo 2 , it suffices to consider the case that $g$ is the Poincaré dual to a section $s$ of $\pi$. (Such a section exists at least over the five-skeleton of $V$, since the Euler class of $N$ is zero, and a choice of $s$ on the five-skeleton suffices for evaluating the fourdimensional class on the left-hand side of $\left({ }_{0} . \overline{2} \overline{0}_{1}^{\prime}\right)$.) Choice of such a section splits $N$ as $N=\mathcal{O} \oplus N^{\prime}$ where $\mathcal{O}$ is a rank one trivial real bundle (consisting of multiples of $s$ ) and $N^{\prime}$ is a rank four bundle. $g \cup g$ is Poincaré dual to the intersection class $s \cap s$. If we regard $s$ as a codimension four submanifold of $P$, then its normal bundle is $N^{\prime}$, so $s \cap s$ is dual to the restriction to $s$ of the Euler class $\chi\left(N^{\prime}\right)$, and hence $\pi_{*}(g \cup g)=\pi_{*}\left(g \cup \chi\left(N^{\prime}\right)\right)=\pi_{*}(g) \cup \chi\left(N^{\prime}\right)=\chi\left(N^{\prime}\right)$. But (for any $\mathrm{SO}(4)$ bundle $N^{\prime}$ ) 
$\chi\left(N^{\prime}\right)$ is congruent to $w_{4}\left(N^{\prime}\right) \bmod 2$, and with $N=\mathcal{O} \oplus N^{\prime}$, we have $w_{4}(N)=w_{4}\left(N^{\prime}\right)$. This justifies the assertion in $(15.201)$.

Pick a class $x \in H^{3}(V ; \mathbb{Z})$. We will now restate the definition of $\Omega(x)=(-1)^{h(x)}$ given in $\left[\begin{array}{l}3 \\ \underline{1}\end{array}\right]$. Let $z=a^{\prime} \cup x \in H^{4}\left(S^{\prime} \times V ; \mathbb{Z}\right)$, with $a^{\prime}$ a generator of $H^{1}\left(S^{\prime} ; \mathbb{Z}\right)$. Let $\widetilde{Z}=S^{\prime} \times P$, where $S^{\prime}$ is a circle with Neveu-Schwarz spin structure. Thus, $\widetilde{Z}$ is a four-sphere bundle over $Z=S^{\prime} \times V$; we write $\widetilde{\pi}$ for the projection $\widetilde{\pi}: \widetilde{Z} \rightarrow Z$. And define $w \in H^{4}(\widetilde{Z} ; \mathbb{Z})$ by $w=\widetilde{\pi}^{*}(z)+g$. Let now $\widetilde{W}$ be a twelve-dimensional spin manifold with boundary $\widetilde{Z}$ over which $w$ extends. Such a $\widetilde{W}$ always exists [i] $\underline{\overline{0}} \overline{]}$. The definition in [3]

$$
h(x)=\frac{1}{3} \int_{\widetilde{W}}\left(w-\frac{1}{2} \lambda\right)\left(\left(w-\frac{1}{2} \lambda\right)^{2}-\frac{1}{8}\left(p_{2}-\lambda^{2}\right)\right)-(w \longrightarrow 0) .
$$

Here the meaning of the last term is that one should subtract the same expression with $w$ replaced by $0 . E_{8}$ index theory is used to prove that $h(x)$ is integral and independent modulo 2 of the choice of $\widetilde{W}$ and of the extension of $w$. The fact that the class that is integrated in ( $(5.2 \overline{1})$ is not canonically trivial near the boundary means that the function $\Omega(x)$ enables us to quantize not the space $H^{3}(V ; \mathrm{U}(1))$ of flat three-form fields on $V$, but a shifted version of it.

The definition of $h(x)$ just given is rather abstract. For computation, it is convenient to make some simplifying assumptions that are actually rather mild in practice. Suppose that $S^{\prime} \times V$ is the boundary of an oriented eight-manifold $W$ over which $N$ extends (as a rank five bundle obeying $w_{1}(N)=0, w_{2}(N)=w_{2}(W)$, and $w_{5}(N)=0$ ). Let $\widetilde{W}$ be the unit sphere bundle in $N$; the conditions on $N$ ensure that $\widetilde{W}$ is spin, and its boundary is $\widetilde{Z}=S^{\prime} \times P$. Suppose further that $z=a^{\prime} \cup x$ extends over $W$,

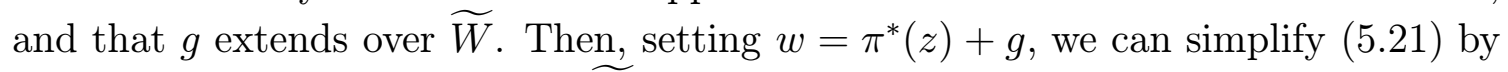
integrating over the fibers of $\pi: \widetilde{W} \rightarrow W$. We get

$$
h(x)=\int_{W}\left(z \cup z-z \cup \lambda(\widetilde{W})+z \cup \pi_{*}(g \cup g)\right) .
$$

(We have dropped terms that vanish if $x=0$; they in fact vanish mod 2 using the fact that the integral in ( $\left(5.2 \overline{1}_{1}^{\prime}\right)$ is even if evaluated on a closed twelve-dimensional spin manifold, and the fact that the integrand vanishes near the boundary if $x=0$.)

To clarify this further, we would like to express the mod 2 reduction of $-\lambda(\widetilde{W})+$ $\pi_{*}(g \cup g)$ in terms of quantities defined just on $W$. For this, we note first that (for any spin manifold $\widetilde{W}) \lambda(\widetilde{W})$ is congruent mod 2 to $w_{4}(\widetilde{W})$. Stably, the tangent bundles $T \widetilde{W}$ and $T W$ of $\widetilde{W}$ and $W$ are related by $T \widetilde{W}=T W \oplus N$. So since $w_{1}(W)=$ $w_{1}(N)=0$ and $w_{2}(N)=w_{2}(W)$, we have $w_{4}(\widetilde{W})=w_{4}(W)+w_{2}(W) w_{2}(N)+w_{4}(N)=$ $w_{4}(W)+w_{2}(W)^{2}+w_{4}(N)$. Using also $\left(\overline{5} . \overline{2} \overline{0}_{1}^{\prime}\right)$, we learn that $-\lambda(\widetilde{W})+\pi_{*}(g \cup g)$ is congruent $\bmod 2$ to $w_{4}(W)+w_{2}(W)^{2}$, so that (15.2.2.

$$
h(x)=\int_{W}\left(z \cup z+w_{4}(W) \cup z+w_{2}(W) \cup w_{2}(W) \cup z\right) .
$$


This is the formula that we guessed on purely formal grounds toward the end of section 1.2. What we have gained is an understanding of how this formula is related to eleven-dimensional physics.

\section{Acknowledgments}

This work was supported in part by NSF Grant PHY-9513835 and the Caltech Discovery Fund. I am grateful to M.J. Hopkins and I.M. Singer for numerous explanations of their viewpoint about the fivebrane action, as well as other matters. In addition, I would like to thank G. Moore for discussions and suggestions about the manuscript, R. Stong for helpful correspondence, and E. Diaconescu, D. Freed, and

A. Kapustin for comments and questions.

\section{References}

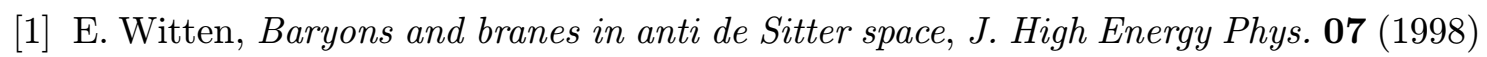
: 006 inep-th/9805112in.

[2] R. Minasian and G. Moore, K-theory and Ramond-Ramond charge, iJ. $\bar{H} \bar{i} \overline{g h}$ Energy

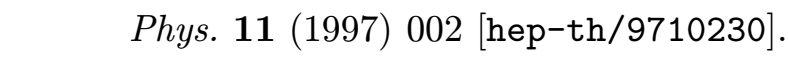

[3] E. Witten, D-branes and K-theory, iJ. High E [hep-th/9810188in.

[4] D.S. Freed and E. Witten, Anomalies in string theory with D-branes, hep-th/9907189.

[5] E. Witten, On flux quantization in M-theory and the effective action, J. Geom. Phys. 22 (1997) 1 [hep-th/9609122in.

[6] N. Marcus and J.H. Schwarz, Field theories that have no manifestly Lorentz invariant

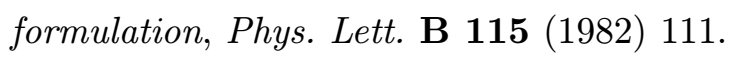

[7] W. Siegel, Manifest Lorentz invariance sometimes requires nonlinearity, 'LB $238(1984) 307$

[8] P. Goddard and D. Olive, Algebras, lattices, and strings, Phys. Scripta T15 (1987).

[9] C. Imbimbo and A. Schwimmer, The lagrangian formulation of chiral scalars, -

[10] C.M. Hull, Covariant quantization of chiral bosons and anomaly cancellation, 'Phys.'. - Lett. B 206 $(1988) 234$

[11] J.M.F. Labastida and M. Pernici, Lagrangians for chiral bosons and the heterotic string, iNucl. Phys. B $\mathbf{3 0 6}(1988) 516$. 
[12] L. Mezincescu and R.I. Nepomechie, Critical dimensions for chiral bosons, 'P (1)

[13] M. Henneaux and C. Teitelboim, Dynamics of chiral (selfdual) p-forms, -

F.P. Devecchi and M. Henneaux, Covariant path integral for chiral p-forms, 'Physys.' : Rev. D 54 (1996) 1606

[14] P.P. Srivastava, On a gauge theory of selfdual field and its quantization, 'Phys. Lett. B. -

[15] B. McClain, F. Yu and Y.S. Wu, Covariant quantization of chiral bosons and $\mathrm{OSp}(1,1 \mid 2)$ symmetry, Nucl. Phys. B $\mathbf{3 4 3}(1990) 689$.

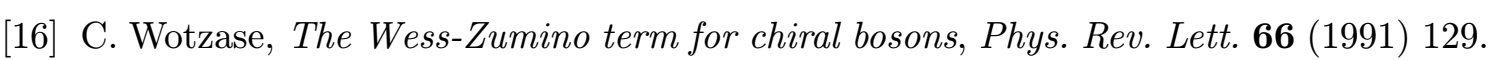

[17] I. Martin and A. Restuccia, Duality symmetric actions and canonical quantization,

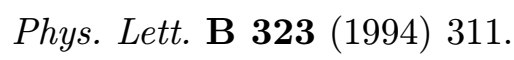

[18] J.H. Schwarz and A. Sen, Duality symmetric actions, 'Lhep-th/930415行.

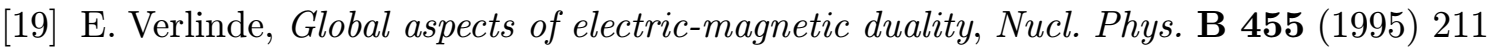
[hep-th/9506011.

[20] M. Perry and J.H. Schwarz, Interacting chiral gauge fields in six dimensions and BornInfeld theory, Noucl. Phys. B 489 (1997) 47 ihep-th/9611065.].

[21] J.H. Schwarz, Coupling a self-dual tensor to gravity in six dimensions, "Phys. Lètt. B. :

[22] N. Berkovits, Local actions with electric and magnetic sources, 'Phys. Lett. (1997) 28 i [hep-th/9610134].

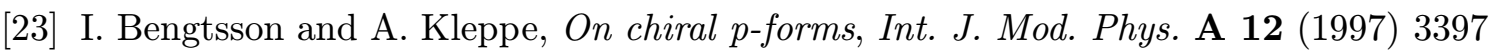
hep-th/9609102in;

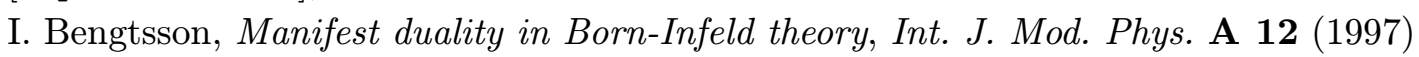
-

[24] P. Pasti, D. Sorokin and M. Tonin, Note on manifest Lorentz and general coordinate invariance in duality symmetric models, iPhys. Lett. B $\mathbf{3 5 2}(1995)$ 59: [hep-th/9503182]; On Lorentz invariant actions for chiral p-forms, Phys. Rev. D [hep-th/9611100,; Covariant action for a $D=11$ five-brane with the chiral field,

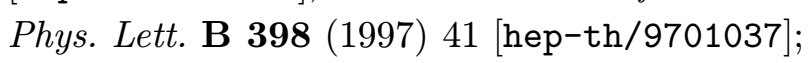

I. Bandos, K. Lechner, A. Nurmagambetov, P. Pasti and D. Sorokin, Covariant action for the super-five-brane of $M$-theory, Phys. Rev. Lett. $\overline{7} \overline{8}(1 \overline{9} \overline{7}) \overline{4} 332$ [hep-th/9701149in;

G. Dall'Agata, K. Lechner and M. Tonin, Action for IIB supergravity in 10 dimensions, hep-th/9812170!. 
[25] M. Aganagic, J. Park, C. Popescu and J.H. Schwarz, World-volume action of the M-theory five-brane, iNucl. Phys. B 496 (1997) 191 ihep-th/9701166̆.

[26] P.S. Howe, E. Sezgin and P.C. West, Covariant field equations of the M-theory five-

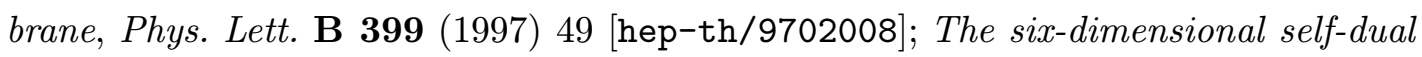

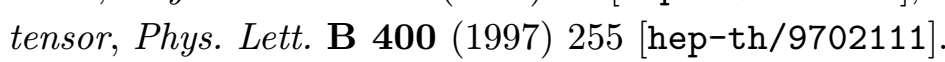

[27] Y.-G. Miao, J.-G. Zhou and Y.-Y. Liu, New way of the derivation of first order wess-

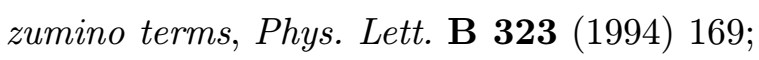

Y.-G. Miao and H.J.W. Müller-Kirsten, Self-duality of various chiral boson actions, hep-th/991206

[28] A. Maznytsia, C.R. Preitschopf and D. Sorokin, Dual actions for chiral bosons, hep-th/9808049.'

[29] X. Bekaert, M. Henneaux and A. Sevrin, Deformations of chiral two-forms in six

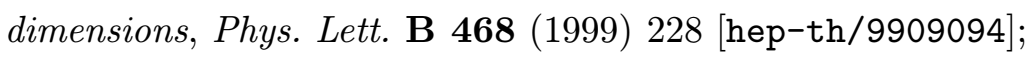

X. Bekaert, M. Henneaux and A. Sevrin, Symmetry-deforming interactions of chiral

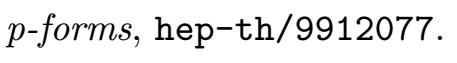

[30] M. Henningson, B.E. W. Nilsson and P. Salomonson, Holomorphic factorization of correlation functions in $(4 k+2)$-dimensional $(2 k)$-form gauge theory, 'J. High Energy,

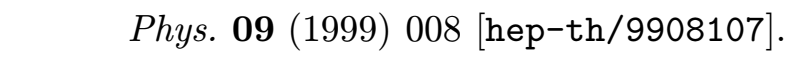

[31] E. Witten, Five-brane effective action in M-theory, J. Geom. Phys. 22 (1997) 103 [hep-th/9610234.

[32] L. Dolan and C.R. Nappi, A modular invariant partition function for the fivebrane, Nucl. Phys. B 530 (1998) 683 [hep-th/9806016].

[33] M. Hopkins and I.M. Singer, to appear.

[34] D.J. Gross, J.A. Harvey, E. Martinec and R. Rohm, The heterotic string, 'P Lett. $5 \overline{4}(1985)-50 \overline{2}$.

[35] D. Freed, J.A. Harvey, R. Minasian and G. Moore, Gravitational anomaly cancellation

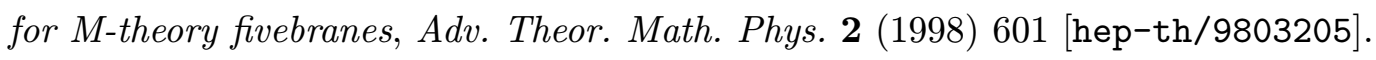

[36] K. Becker and M. Becker, Fivebrane gravitational anomalies, hep-th/9911138i.

[37] E. Witten, On S-duality in abelian gauge theory, hep-th/9505186i.

[38] M.F. Atiyah and I.M. Singer, The index of elliptic operators, 5, Ann. Math. 93 (1971) 139.

[39] M.F. Atiyah, K-theory and reality, Quart. J. Math. Oxford (2) 17 (1966) 367.

[40] R. Stong, Calculation of $\Omega_{11}^{\mathrm{spin}}(K(\mathbb{Z}, 4)$, in Unified string theories,M.B. Green and D.J. Gross eds., World Scientific, 1986. 